\title{
On the Number of Planar Orientations with Prescribed Degrees*
}

\author{
Stefan Felsner \\ Florian Zickfeld \\ Technische Universität Berlin, Fachbereich Mathematik \\ Straße des 17. Juni 136, 10623 Berlin, Germany \\ \{felsner, zickfeld\}@math.tu-berlin.de
}

Submitted: Sep 6, 2007; Accepted: May 27, 2008; Published: Jun 6, 2008

\begin{abstract}
We deal with the asymptotic enumeration of combinatorial structures on planar maps. Prominent instances of such problems are the enumeration of spanning trees, bipartite perfect matchings, and ice models. The notion of orientations with outdegrees prescribed by a function $\alpha: V \rightarrow \mathbb{N}$ unifies many different combinatorial structures, including the afore mentioned. We call these orientations $\alpha$-orientations. The main focus of this paper are bounds for the maximum number of $\alpha$-orientations that a planar map with $n$ vertices can have, for different instances of $\alpha$. We give examples of triangulations with $2.37^{n}$ Schnyder woods, 3-connected planar maps with $3.209^{n}$ Schnyder woods and inner triangulations with $2.91^{n}$ bipolar orientations. These lower bounds are accompanied by upper bounds of $3.56^{n}, 8^{n}$ and $3.97^{n}$ respectively. We also show that for any planar map $M$ and any $\alpha$ the number of $\alpha$-orientations is bounded from above by $3.73^{n}$ and describe a family of maps which have at least $2.598^{n} \alpha$-orientations.

AMS Math Subject Classification: 05A16, 05C20, 05C30
\end{abstract}

\section{Introduction}

A planar map is a planar graph together with a crossing-free drawing in the plane. Many different structures on connected planar maps have attracted the attention of researchers. Among them are spanning trees, bipartite perfect matchings (or more generally bipartite $f$-factors), Eulerian orientations, Schnyder woods, bipolar orientations and 2-orientations of quadrangulations. The concept of orientations with prescribed out-degrees is a quite

${ }^{*}$ The conference version of this paper has appeared in the proceedings of WG'07 (LNCS 4769, pp. 190-201) under the title "On the Number of $\alpha$-Orientations". 
general one. Remarkably, all the above structures can be encoded as orientations with prescribed out-degrees. Let a planar map $M$ with vertex set $V$ and a function $\alpha: V \rightarrow \mathbb{N}$ be given. An orientation $X$ of the edges of $M$ is an $\alpha$-orientation if every vertex $v$ has outdegree $\alpha(v)$. For the sake of brevity, we refer to orientations with prescribed out-degrees simply as $\alpha$-orientations in this paper.

For some of the above mentioned structures it is not obvious how to encode them as $\alpha$-orientations. For Schnyder woods on triangulations the encoding by 3-orientations goes back to de Fraysseix and de Mendez [10]. For bipolar orientations an encoding was proposed by Woods [40] and independently by Tamassia and Tollis [35]. Bipolar orientations of $M$ are one of the structures which cannot be encoded as $\alpha$-orientations on $M$, an auxiliary map $M^{\prime}$ (the angle graph of $M$ ) has to be used instead. For Schnyder woods on 3-connected planar maps as well as bipartite $f$-factors and spanning trees Felsner [14] describes encodings as $\alpha$-orientations. He also proves that the set of $\alpha$-orientations of a planar map $M$ can always be endowed with the structure of a distributive lattice. This structure on the set of $\alpha$-orientations found applications in drawing algorithms in [4], [17], and for enumeration and random sampling of graphs in [19].

Given the existence of a combinatorial structure on a class $\mathcal{M}_{n}$ of planar maps with $n$ vertices, one of the questions of interest is how many such structures there are for a given map $M \in \mathcal{M}_{n}$. Especially, one is interested in the minimum and maximum that this number attains on the maps from $\mathcal{M}_{n}$. This question has been treated quite successfully for spanning trees and bipartite perfect matchings. For spanning trees the Kirchhoff Matrix Tree Theorem allows to bound the maximum number of spanning trees of a planar graph with $n$ vertices between $5.02^{n}$ and $5.34^{n}$, see $[31,28]$. Pfaffian orientations can be used to efficiently calculate the number of bipartite perfect matchings in the planar case, see for example [24]. Kasteleyn has shown, that the $k \times \ell$ square grid has asymptotically $e^{0.29 \cdot k \ell} \approx 1.34^{k \ell}$ perfect matchings. The number of Eulerian orientations is studied in statistical physics under the name of ice models, see [2] for an overview. In particular Lieb [22] has shown that the square grid on the torus has asymptotically $(8 \sqrt{3} / 9)^{k \ell} \approx 1.53^{k \ell}$ Eulerian orientations and Baxter [1] has worked out the asymptotics for the triangular grid on the torus as $(3 \sqrt{3} / 2)^{k \ell} \approx 2.598^{k \ell}$.

In many cases it is relatively easy to see which maps in a class $\mathcal{M}_{n}$ carry a unique object of a certain type, while the question about the maximum number is rather intricate. Therefore, we focus on finding the asymptotics for the maximum number of $\alpha$-orientations that a map from $\mathcal{M}_{n}$ can carry. The next table gives an overview of the results of this paper for different instances of $\mathcal{M}_{n}$ and $\alpha$. The entry $c$ in the "Upper Bound" column is to be read as $O\left(c^{n}\right)$, in the "Lower Bound" column as $\Omega\left(c^{n}\right)$ and for the " $\approx c$ " entries the asymptotics are known.

The paper is organized as follows. In Section 2 we treat the most general case, where $\mathcal{M}_{n}$ is the class of all planar maps with $n$ vertices and $\alpha$ can be any integer valued function. We prove an upper bound which applies for every map and every $\alpha$. In Section 2.3 we deal with Eulerian orientations. In Section 3.1 we consider Schnyder woods on plane triangulations and in Section 3.2 the more general case of Schnyder woods on 3-connected planar maps. We split the treatment of Schnyder woods because the more 


\begin{tabular}{|l||c|c|}
\hline \multicolumn{1}{|c||}{ Graph class and orientation type } & Lower bound & Upper bound \\
\hline$\alpha$-orientations on planar maps & 2.598 & 3.73 \\
\hline Eulerian orientations & 2.598 & 3.73 \\
\hline Schnyder woods on triangulations & 2.37 & 3.56 \\
\hline Schnyder woods on the square grid & \multicolumn{2}{c|}{$\approx 3.209$} \\
\hline Schnyder woods on 3-connected planar maps & 1.53 & 8 \\
\hline 2-orientations on quadrangulations & \multicolumn{2}{|c|}{$\approx 2$} \\
\hline bipolar orient. on stacked triangulations & \multicolumn{2}{c|}{$\approx 1.618$} \\
\hline bipolar orientations on outerplanar maps & 2.18 & 2.62 \\
\hline bipolar orientations on the square grid & 2.91 & 3.97 \\
\hline bipolar orientations on planar maps & \multicolumn{2}{|c|}{} \\
\hline
\end{tabular}

direct encoding of Schnyder woods on triangulations as $\alpha$-orientations yields stronger bounds. In Section 3.2 we also discuss the asymptotic number of Schnyder woods on the square grid. Section 4 is dedicated to 2-orientations of quadrangulations. In Section 5, we study bipolar orientations on the square grid, stacked triangulations, outerplanar maps and planar maps. The upper bound for planar maps relies on a new encoding of bipolar orientations of inner triangulations. In Section 6.1 we discuss the complexity of counting $\alpha$-orientations. In Section 6.2 we show how counting $\alpha$-orientations can be reduced to counting (not necessarily planar) bipartite perfect matchings and the consequences of this connection are discussed as well. We conclude with some open problems.

\section{Counting $\alpha$-Orientations}

A planar map $M$ is a simple planar graph $G$ together with a fixed crossing-free embedding of $G$ in the Euclidean plane. In particular, $M$ has a designated outer (unbounded) face. We denote the sets of vertices, edges and faces of a given planar map by $V, E$, and $\mathcal{F}$, and their respective cardinalities by $n, m$ and $f$. The degree of a vertex $v$ will be denoted by $d(v)$.

Let $M$ be a planar map and $\alpha: V \rightarrow \mathbb{N}$. An orientation $X$ of the edges of $M$ is an $\alpha$-orientation if for all $v \in V$ exactly $\alpha(v)$ edges are directed away from $v$ in $X$.

Let $X$ be an $\alpha$-orientation of $M$ and let $C$ be a directed cycle in $X$. Define $X^{C}$ as the orientation obtained from $X$ by reversing all edges of $C$. Since the reversal of a directed cycle does not affect out-degrees the orientation $X^{C}$ is also an $\alpha$-orientation of $M$. The plane embedding of $M$ allows us to classify a directed simple cycle as clockwise (cw-cycle) if the interior, $\operatorname{Int}(C)$, is to the right of $C$ or as counterclockwise (ccw-cycle) if $\operatorname{lnt}(C)$ is to the left of $C$. If $C$ is a ccw-cycle of $X$ then we say that $X^{C}$ is left of $X$ and $X$ is right of $X^{C}$. Felsner proved the following theorem in [14].

planar map $\alpha$-orientation cw-cycle ccw-cycle left of right of 
Theorem 1 Let $M$ be a planar map and $\alpha: V \rightarrow \mathbb{N}$. The set of $\alpha$-orientations of $M$ endowed with the transitive closure of the 'left of' relation is a distributive lattice.

The following observation is easy, but useful. Let $M$ and $\alpha: V \rightarrow \mathbb{N}$ be given, $W \subset V$ and $E_{W}$ the edges of $M$ with one endpoint in $W$ and the other endpoint in $V \backslash W$. Suppose all edges of $E_{W}$ are directed away from $W$ in some $\alpha$-orientation $X_{0}$ of $M$. The demand of $W$ for $\sum_{w \in W} \alpha(w)$ outgoing edges forces all edges in $E_{W}$ to be directed away from $W$ in every $\alpha$-orientation of $M$. Such an edge with the same direction in every $\alpha$-orientation is a rigid edge.

We denote the number of $\alpha$-orientations of $M$ by $r_{\alpha}(M)$. Let $\mathcal{M}$ be a family of pairs rigid edge $(M, \alpha)$ of a planar map and an out-degree function. Most of this paper is concerned with lower and upper bounds for $\max _{(M, \alpha) \in \mathcal{M}} r_{\alpha}(M)$ for some family $\mathcal{M}$. In Section 2.1, we deal with bounds which apply to all $M$ and $\alpha$, while later sections will be concerned with special instances.

\subsection{An Upper Bound for the Number $\alpha$-Orientations}

A trivial upper bound for the number of $\alpha$-orientations on $M$ is $2^{m}$ as any edge can be directed in two ways. The following easy but useful lemma improves the trivial bound.

Lemma 1 Let $M$ be a planar map, $A \subset E$ a cycle free subset of edges of $M$, and $\alpha$ a function $\alpha: V \rightarrow \mathbb{N}$. Then, there are at most $2^{m-|A|} \alpha$-orientations of $M$. Furthermore, $M$ has less than $4^{n} \alpha$-orientations.

Proof. Let $X$ be an arbitrary but fixed orientation out of the $2^{m-|A|}$ orientations of the edges of $E \backslash A$. It suffices to show that $X$ can be extended to an $\alpha$-orientation of $M$ in at most one way. We proceed by induction on $|A|$. The base case $|A|=0$ is trivial. If $|A|>0$, then, as $A$ is cycle free, there is a vertex $v$, which is incident to exactly one edge $e \in A$. If $v$ has out-degree $\alpha(v)$ respectively $\alpha(v)-1$ in $X$, then $e$ must be directed towards respectively away from $v$. In either case the direction of $e$ is determined by $X$, and by induction there is at most one way to extend the resulting orientation of $E \backslash(A-e)$ to an $\alpha$-orientation of $M$. If $v$ does not have out-degree $\alpha(v)$ or $\alpha(v)-1$ in $X$, then there is no extension of $X$ to an $\alpha$-orientation of $M$. The bound $2^{m-n+1}<4^{n}$ follows by choosing $A$ to be a spanning forest and applying Euler's formula.

A better upper bound for general $M$ and $\alpha$ will be given in Proposition 1 . The following lemma is needed for the proof.

Lemma 2 Let $M$ be a planar map with $n$ vertices that has an independent set $I_{2}$ of $n_{2}$ vertices which have degree 2 in $M$. Then, $M$ has at most $(3 n-6)-\left(n_{2}-1\right)$ edges.

Proof. Consider a triangulation $T$ extending $M$ and let $B$ be the set of additional edges, i.e., of edges of $T$ which are not in $M$. If $n=3$ the conclusion of the lemma is true and we may thus assume $n>3$ for the rest of the proof. Hence, there are no vertices of degree 2 in $T$, and every vertex of $I_{2}$ must be incident to at least one edge from $B$. If there is a vertex $v \in I_{2}$, which is incident to exactly one edge from $B$, then $v$ and its incident edges 
can be deleted from $I_{2}$, from $M$ and from $T$, whereby the result follows by induction. The last case is that all vertices of $I_{2}$ have at least two incident edges in $B$. Since every edge in $B$ is incident to at most two vertices from $I_{2}$ it follows that $\left|I_{2}\right| \leq|B|$. Therefore, $\left|E_{M}\right|=\left|E_{T}\right|-|B| \leq\left|E_{T}\right|-\left|I_{2}\right|=(3 n-6)-n_{2}$.

Remark. It can be seen from the above proof, that $K_{2, n_{2}}$ plus the edge between the two vertices of degree $n_{2}$ is the unique graph to which only $n_{2}-1$ edges can be added. For every other graph at least $n_{2}$ edges can be added.

Proposition 1 Let $M$ be a planar map, $\alpha: V \rightarrow \mathbb{N}$, and $I=I_{1} \cup I_{2}$ an independent set of $M$, where $I_{2}$ is the subset of degree 2 vertices in $I$. Then, $M$ has at most

$$
2^{2 n-4-\left|I_{2}\right|} \cdot \prod_{v \in I_{1}}\left(\frac{1}{2^{d(v)-1}}\left(\begin{array}{l}
d(v) \\
\alpha(v)
\end{array}\right)\right)
$$

$\alpha$-orientations.

Proof. We may assume that $M$ is connected. Let $M_{i}$, for $i=1, \ldots c$, be the components of $M-I$. We claim that $M$ has at most $(3 n-6)-(c-1)-\left(\left|I_{2}\right|-1\right)$ edges. Note, that every component $C$ of $M-I$ must be connected to some other component $C^{\prime}$ via a vertex $v \in I$ such that the edges $v w$ and $v w^{\prime}$ with $w \in C$ and $w^{\prime} \in C^{\prime}$ form an angle at $v$. As $w$ and $w^{\prime}$ are in different connected components the edge $w w^{\prime}$ is not in $M$ and we can add it without destroying planarity. We can add at least $c-1$ edges not incident to $I$ in this fashion. Thus, by Lemma 2 we have that $m+(c-1) \leq 3 n-6-\left(I_{2}-1\right)$.

Let $S^{\prime}$ be a spanning forest of $M-I$, and let $S$ be obtained from $S^{\prime}$ by adding one edge incident to every $v \in I$. Then, $S$ is a forest with $n-c$ edges. By Lemma $1 M$ has at most $2^{m-|S|} \alpha$-orientations and by Lemma 2

$$
m-|S| \leq(3 n-6)-(c-1)-\left(\left|I_{2}\right|-1\right)-(n-c)=2 n-4-\left|I_{2}\right| .
$$

For every vertex $v \in I_{1}$ there are $2^{d(v)-1}$ possible orientations of the edges of $M-S$ at $v$. Only the orientations with $\alpha(v)$ or $\alpha(v)-1$ outgoing edges at $v$ can potentially be completed to an $\alpha$-orientation of $M$. Since $I_{1}$ is an independent set it follows that $M$ has at most

$$
2^{m-|S|} \cdot \prod_{v \in I_{1}} \frac{1}{2^{d(v)-1}}\left(\left(\begin{array}{c}
d(v)-1 \\
\alpha(v)
\end{array}\right)+\left(\begin{array}{c}
d(v)-1 \\
\alpha(v)-1
\end{array}\right)\right) \leq 2^{2 n-4-\left|I_{2}\right|} \cdot \prod_{v \in I_{1}}\left(\frac{1}{2^{d(v)-1}}\left(\begin{array}{c}
d(v) \\
\alpha(v)
\end{array}\right)\right)
$$

$\alpha$-orientations.

Corollary 1 Let $M$ be a planar map and $\alpha: V \rightarrow \mathbb{N}$. Then, $M$ has at most $3.73^{n}$ $\alpha$-orientations.

Proof. Since $M$ is planar the Four Color Theorem implies, that it has an independent set $I$ of size $|I| \geq n / 4$. Let $I_{1}, I_{2}$ be as above. Note, that for $d(v) \geq 3$

$$
\frac{1}{2^{d(v)-1}}\left(\begin{array}{c}
d(v) \\
\alpha(v)
\end{array}\right) \leq \frac{1}{2^{d(v)-1}}\left(\begin{array}{c}
d(v) \\
\lfloor(v) / 2\rfloor
\end{array}\right) \leq \frac{3}{4} .
$$


Thus, the result follows from Proposition 1, as

$$
2^{2 n-4-\left|I_{2}\right|}\left(\frac{3}{4}\right)^{\left|I_{1}\right|} \leq 2^{2 n-4}\left(\frac{3}{4}\right)^{\frac{n}{4}} \leq 3.73^{n} .
$$

Remark. The best lower bound for general $\alpha$ and $M$, which we can prove, comes from Eulerian orientations of the triangular grid, see Section 2.3 .

\subsection{Grid Graphs}

Enumeration and counting of different combinatorial structures on grid graphs have received a lot of attention in the literature, see e.g. [2, 7, 22]. In Section 2.3 we present a family of graphs that have asymptotically at least $2.598^{n}$ Eulerian orientations. This family is closely related to the grid graph, and throughout the paper we will use different relatives of the grid graph to obtain lower bounds. We collect the definitions of these related families here.

The grid graph $G_{k, \ell}$ with $k$ rows and $\ell$ columns is defined as follows. The vertex set is

$$
V_{k, \ell}=\{(i, j) \mid 1 \leq i \leq k, 1 \leq j \leq \ell\} .
$$

The edge set $E_{k, \ell}=E_{k, \ell}^{H} \cup E_{k, \ell}^{V}$ consists of horizontal edges

$$
E_{k, \ell}^{H}=\{\{(i, j),(i, j+1)\} \mid 1 \leq i \leq k, 1 \leq j \leq \ell-1\}
$$

and vertical edges

$$
E_{k, \ell}^{V}=\{\{(i, j),(i+1, j)\} \mid 1 \leq i \leq k-1,1 \leq j \leq \ell\} .
$$

We denote the $i$ th vertex row by $V_{i}^{R}=\{(i, j) \mid 1 \leq j \leq \ell\}$ and the $j$ th vertex column by $V_{j}^{C}=\{(i, j) \mid 1 \leq i \leq k\}$. The $j$ th edge column $E_{j}^{C}$ is defined as $E_{j}^{C}=\{\{(i, j),(i, j+1)\} \mid$ $1 \leq i \leq k\}$. The number of bipolar orientations of $G_{k, \ell}$ is studied in Section 5.1.

The grid on the torus $G_{k, \ell}^{T}$ is obtained from $G_{k+1, \ell+1}$ by identifying $(1, i)$ and $(k+$ $1, i)$ as well as $(j, 1)$ and $(j, \ell+1)$ for all $i$ and $j$, see Figures 1 (a) and (b). Edges of the form $\{(i, 1),(i, \ell)\}$ are called horizontal wrap-around edges while those of the form $\{(1, j),(k, j)\}$ are the vertical wrap-around edges. Note that $G_{k, \ell}$ can be obtained from $G_{k, \ell}^{T}$ by deleting the $k$ horizontal and the $\ell$ vertical wrap-around edges.

Lieb [22] shows that $G_{k, \ell}^{T}$ has asymptotically $(8 \sqrt{3} / 9)^{k \ell}$ Eulerian orientations. His analysis involves the calculation of the dominant eigenvalue of a so-called transfer matrix, see also Section 4.

We consider the number of Schnyder woods on the augmented grid $G_{k, \ell}^{*}$ in Section 3.2, see Figure 1 (c). The augmented grid is obtained from $G_{k, \ell}$ by adding a triangle with vertices $\left\{a_{1}, a_{2}, a_{3}\right\}$ to the outer face. The triangle is connected to the boundary vertices 
(a)

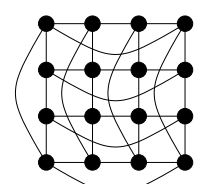

(b)

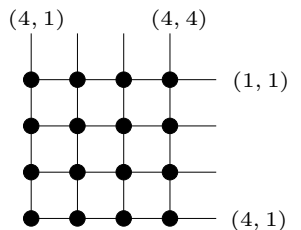

(c)

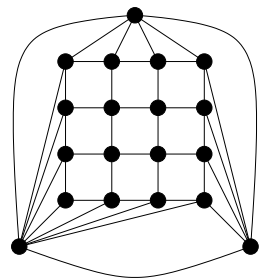

(d)

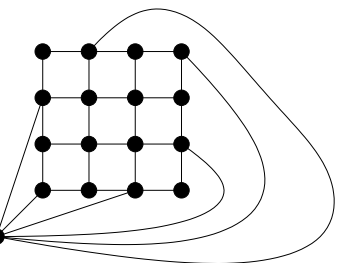

Figure 1: Two illustrations of $G_{4,4}^{T}$, the augmented grid $G_{4,4}^{*}$, and the quadrangulation $G_{4,4}^{\square}$.

of the grid as follows. The vertex $a_{1}$ is adjacent to all vertices of $V_{1}^{R}, a_{2}$ is adjacent the vertices from $V_{\ell}^{C}$ and $a_{3}$ to the vertices from $V_{k}^{R} \cup V_{1}^{C}$.

When we consider 2-orientations in Section 4 we use the quadrangulation $G_{k, \ell}^{\square}$, see Figure 1 (d). It is obtained from the grid $G_{k, \ell}$ by adding one vertex $v_{\infty}$ to the outer face which is adjacent to every other vertex of the boundary such that $(1,1)$ is not adjacent to $v_{\infty}$. For $k$ and $\ell$ even this graph is closely related to the torus grid $G_{k, \ell}^{T}$, which can be obtained from $G_{k, \ell}^{\square}$ by reassigning end vertices of edge as follows.

$$
\begin{array}{llll}
\left\{(1, j), v_{\infty}\right\} \rightarrow\{(1, j),(k, j)\} & 2 \leq j \leq \ell & \left\{(k, j), v_{\infty}\right\} \rightarrow\{(k, j),(1, j)\} & 2 \leq j \leq \ell \\
\left\{(i, 1), v_{\infty}\right\} \rightarrow\{(i, 1),(i, \ell)\} & 2 \leq i \leq k & \left\{(i, \ell), v_{\infty}\right\} \rightarrow\{(i, \ell),(i, 1)\} & 2 \leq i \leq k
\end{array}
$$

Since $k, \ell$ are even this does not create parallel edges and the resulting graph is $G_{k, \ell}^{T}$ minus the edges $e_{1}=\{(1,1),(1, \ell)\}$ and $e_{2}=\{(1,1),(k, 1)\}$.

We also use the triangular grid $T_{k, \ell}$ in Sections 2.3 and 5.2. It is obtained from $G_{k, \ell}$ by adding the diagonal edges $\{(i, j),(i-1, j+1)\}$ for $2 \leq i \leq k$ and $1 \leq j \leq \ell-1$, see Figure 2 (a). The augmented triangular grid $T_{k, \ell}^{*}$, which we need in Section 3.1 is obtained in the same way from $G_{k, \ell}^{*}$, see Figure 7 .

The terms vertex row, vertex column and edge column are used for the triangular grid analogously to the definition above for $G_{k, \ell}$.

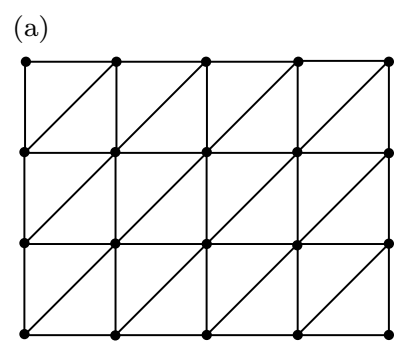

$T_{4,5}$ (b)

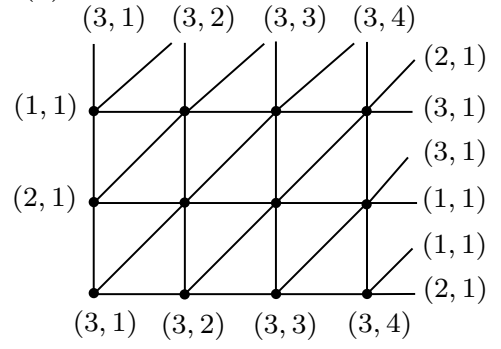

$T_{3,4}^{T}$

Figure 2: The triangular grid $T_{4,5}$, and $T_{3,4}^{T}$.

We also use the triangular grid on the torus $T_{k, \ell}^{T}$, see Figure $2(\mathrm{~b})$. We adopt the definition from [1], therefore it differs slightly from that of the square grid on the torus. 
More precisely, instead of identifying vertices $(i, \ell+1)$ and $(i, 1)$ we identify vertices $(i, \ell+1)$ and $(i-1,1)$ (and $(1, \ell+1)$ with $(k, 1)$ ) to obtain $T_{k, \ell}^{T}$ from $T_{k, \ell}$. This boundary condition is called helical. The wrap-around edges are defined analogously to the square grid case.

Baxter [1] was able to determine the exponential growth factor of Eulerian orientations of $T_{k, \ell}^{T}$ as $k, \ell \rightarrow \infty$. Baxter's analysis uses similar techniques as Lieb's [22] and yields an asymptotic growth rate of $(3 \sqrt{3} / 2)^{k \ell}$.

\subsection{A Lower Bound Using Eulerian Orientations}

Let $M$ be a planar map such that every $v \in V$ has even degree and let $\alpha$ be defined as $\alpha(v)=d(v) / 2, \forall v \in V$. The corresponding $\alpha$-orientations of $M$ are known as Eulerian orientations. Eulerian orientations are exactly the orientations which maximize the binomial coefficients in equation (1). The lower bound in the next theorem is the best lower bound we have for $\max _{(M, \alpha) \in \mathcal{M}} r_{\alpha}(M)$, where $\mathcal{M}$ is the set of all planar maps and no

Eulerian orientarestrictions are made for $\alpha$.

Theorem 2 Let $\mathcal{M}_{n}$ denote the set of all planar maps with $n$ vertices and $\mathcal{E}(M)$ the set of Eulerian orientations of $M \in \mathcal{M}_{n}$. Then, for $n$ big enough,

$$
2.59^{n} \leq(3 \sqrt{3} / 2)^{k \ell} \leq \max _{M \in \mathcal{M}_{n}}|\mathcal{E}(M)| \leq 3.73^{n}
$$

Proof. The upper bound is the one from Corollary 1. For the lower bound consider the triangular torus grid $T_{k, \ell}^{T}$. As mentioned above Baxter [1] was able to determine the exponential growth factor of Eulerian orientations of $T_{k, \ell}^{T}$ as $k, \ell \rightarrow \infty$. Baxter's analysis uses eigenvector calculations and yields an asymptotic growth rate of $(3 \sqrt{3} / 2)^{k \ell}$. This graph can be made into a planar map $T_{k, \ell}^{+}$by introducing a new vertex $v_{\infty}$ which is incident to all the wrap-around edges. This way all crossings between wrap-around edges can be substituted by $v_{\infty}$. As every Eulerian orientation of $T_{k, \ell}^{T}$ yields a Eulerian orientation of $T_{k, \ell}^{+}$this graph has at least $(3 \sqrt{3} / 2)^{k \ell} \geq 2.598^{k \ell}$ Eulerian orientations for $k, \ell$ big enough.

\section{Counting Schnyder Woods}

Schnyder woods for triangulations have been introduced as a tool for graph drawing and graph dimension theory in $[32,33]$. Schnyder woods for 3-connected planar maps are introduced in [12]. Here we review the definition of Schnyder woods and explain how they are encoded as $\alpha$-orientations. For a comprehensive introduction see e.g. [13].

Let $M$ be a planar map with three vertices $a_{1}, a_{2}, a_{3}$ occurring in clockwise order on the outer face of $M$. A suspension $M^{\sigma}$ of $M$ is obtained by attaching a half-edge that reaches into the outer face to each of these special vertices.

Let $M^{\sigma}$ be a suspended 3-connected planar map. A Schnyder wood rooted at $a_{1}, a_{2}, a_{3}$ 
is an orientation and coloring of the edges of $M^{\sigma}$ with the colors 1,2,3 satisfying the following rules.

(W1) Every edge $e$ is oriented in one direction or in two opposite directions. The directions of edges are colored such that if $e$ is bidirected the two directions have distinct colors.

(W2) The half-edge at $a_{i}$ is directed outwards and has color $i$.

(W3) Every vertex $v$ has out-degree one in each color. The edges $e_{1}, e_{2}, e_{3}$ leaving $v$ in colors 1,2,3 occur in clockwise order. Each edge entering $v$ in color $i$ enters $v$ in the clockwise sector from $e_{i+1}$ to $e_{i-1}$, see Figure 3 (a).

(W4) There is no interior face the boundary of which is a monochromatic directed cycle.

(a)

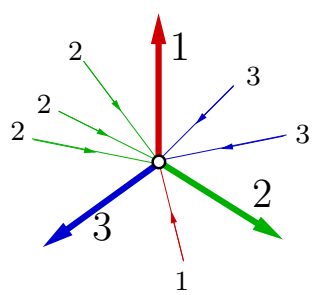

(b)

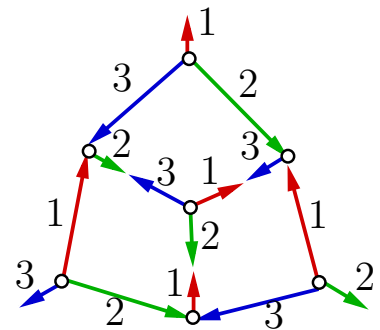

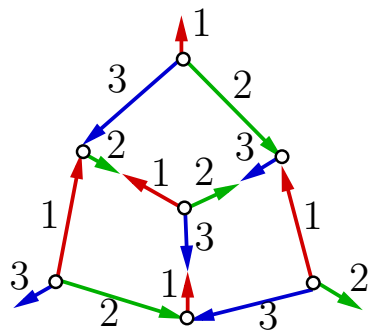

Figure 3: The left part shows edge orientations and edge colors at a vertex, the right part two different Schnyder woods with the same underlying orientation.

In the context of this paper the choice of the suspension vertices is not important and we refer to the Schnyder wood of a planar map, without specifying the suspension explicitly.

Let $M^{\sigma}$ be a planar map with a Schnyder wood. Let $T_{i}$ denote the digraph induced by the directed edges of color $i$. Every inner vertex has out-degree one in $T_{i}$ and in fact $T_{i}$ is a directed spanning tree of $M$ with root $a_{i}$.

In a Schnyder wood on a triangulation only the three outer edges are bidirected. This is because the three spanning trees have to cover all $3 n-6$ edges of the triangulation and the edges of the outer triangle must be bidirected because of the rule of vertices. Theorem 3 says, that the edge orientations together with the colors of the special vertices are sufficient to encode a Schnyder wood on a triangulation, the edge colors can be deduced, for a proof see $[10]$.

Theorem 3 Let $T$ be a plane triangulation, with vertices $a_{1}, a_{2}, a_{3}$ occuring in clockwise order on the outer face. Let $\alpha_{T}(v):=3$ if $v$ is an internal vertex and $\alpha_{T}\left(a_{i}\right):=0$ for $i=1,2,3$. Then, there is a bijection between the Schnyder woods of $T$ and the $\alpha_{T^{-}}$ orientations of the inner edges of $T$.

In the sequel we refer to an $\alpha_{T}$-orientation simply as a 3-orientation. Schnyder woods on 3-connected planar maps are in general not uniquely determined by the edge orientations, see Figure 3 (b). Nevertheless, there is a bijection between the Schnyder woods 
of a 3-connected planar map $M$ and certain $\alpha$-orientations on a related planar map $\widetilde{M}$, see [14].

In order to describe the bijection precisely, we first define the suspension dual $M^{\sigma^{*}}$ of $M^{\sigma}$, which is obtained from the dual $M^{*}$ of $M$ as follows. Replace the vertex $v_{\infty}^{*}$, which represents the unbounded face of $M$ in $M^{*}$, by a triangle on three new vertices $b_{1}, b_{2}, b_{3}$. Let $P_{i}$ be the path from $a_{i-1}$ to $a_{i+1}$ on the outer face of $M$ which avoids $a_{i}$. In $M^{\sigma^{*}}$ the sion dual edges dual to those on $P_{i}$ are incident to $b_{i}$ instead of $v_{\infty}^{*}$. Adding a ray to each of the $b_{i}$ yields $M^{\sigma^{*}}$. An example is given in Figure 4 .
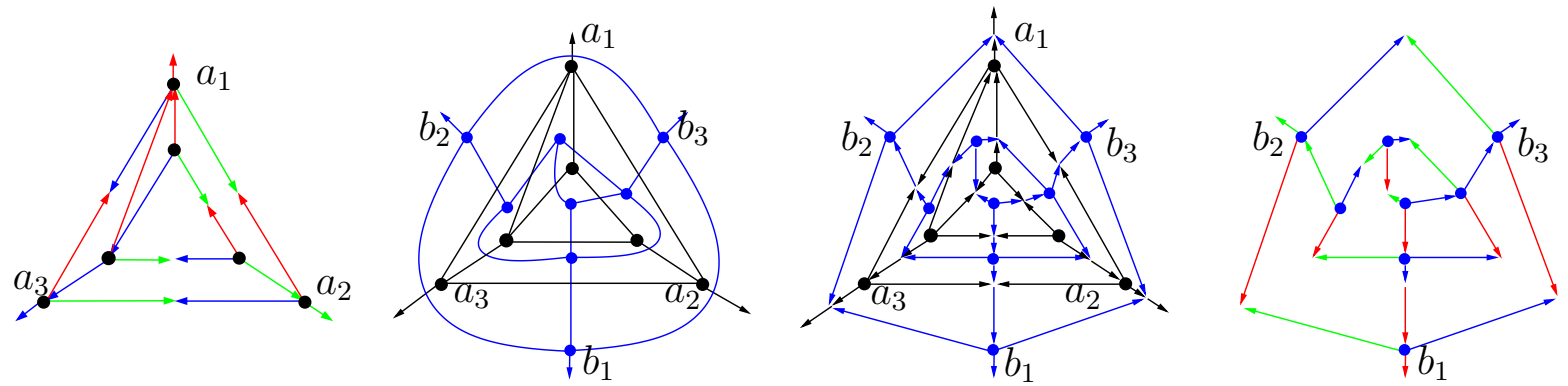

Figure 4: A Schnyder wood, the primal and the dual graph, the oriented primal dual completion and the dual Schnyder wood.

Proposition 2 Let $M^{\sigma}$ be a suspended planar map. There is a bijection between the Schnyder woods of $M^{\sigma}$ and the Schnyder woods of the suspension dual $M^{\sigma^{*}}$. Figure 5 illustrates how the coloring and orientation of a pair of a primal and a dual edge are related.
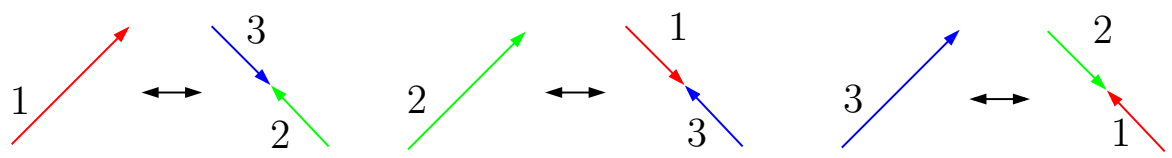

Figure 5: The three possible oriented colorings of a pair of a primal and a dual edge.

The completion $\widetilde{M}$ of $M^{\sigma}$ and $M^{\sigma^{*}}$ is obtained by superimposing the two graphs such that exactly the primal dual pairs of edges cross, see Figure 4 . In the completion $\widetilde{M}$ the common subdivision of each crossing pair of edges is replaced by a new edge-vertex. Note that the rays emanating from the three special vertices of $M^{\sigma}$ cross the three edges of the triangle induced by $b_{1}, b_{2}, b_{3}$ and thus produce edge vertices. The six rays emanating into the unbounded face of the completion end at a new vertex $v_{\infty}$ placed in this unbounded face. A pair of corresponding Schnyder woods on $M^{\sigma}$ and $M^{\sigma^{*}}$ induces an orientation of $\widetilde{M}$ which is an $\alpha_{S^{-}}$orientation where

$$
\alpha_{S}(v)= \begin{cases}3 & \text { for primal and dual vertices } \\ 1 & \text { for edge vertices } \\ 0 & \text { for } v_{\infty} .\end{cases}
$$


Note, that a pair of a primal and a dual edge always consists of a unidirected and a bidirected edge, which explains why $\alpha_{S}\left(v_{e}\right)=1$ is the right choice. Theorem 4 says, that the edge orientations of $\widetilde{M}$ are sufficient to encode a Schnyder wood of $M^{\sigma}$, the edge colors can be deduced, for a proof see [14].

Theorem 4 The Schnyder woods of a suspended planar map $M^{\sigma}$ are in bijection with the $\alpha_{S}$-orientations of $\bar{M}$.

In the rest of this section we give asymptotic bounds for the maximum number of Schnyder woods on planar triangulations and 3-connected planar maps. We treat these two classes separately because the more direct bijection from Theorem 3 allows us to obtain a better upper bound for Schnyder woods on triangulations than for the general case. We also have a better lower bound for the general case of Schnyder woods on 3-connected planar maps than for the restriction to triangulations.

Stacked triangulations are plane triangulations which can be obtained from a triangle by iteratively adding vertices of degree 3 into bounded faces. The stacked triangulations are exactly the plane triangulations which have a unique Schnyder wood and we have a generalization of this well-known result for general 3-connected planar maps, which we state here without a proof.

Theorem 5 All 3-connected planar maps, which have a unique Schnyder wood, can be constructed from the unique Schnyder wood on the triangle by the six operations show in Figure 6 read from left to right.
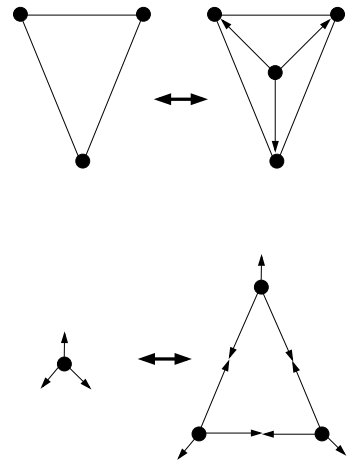
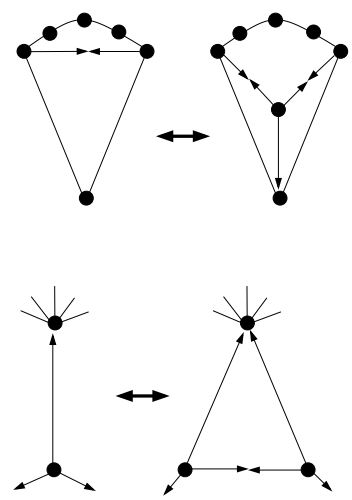

Stacked triangulations

Figure 6: Using the three primal operations in the first row and their duals in the second row every graph with a unique Schnyder wood can be constructed.

\subsection{Schnyder Woods on Triangulations}

Bonichon [3] found a bijection between Schnyder woods on triangulations with $n$ vertices and pairs of non-crossing Dyck-paths, which implies that there are $C_{n+2} C_{n}-C_{n+1}^{2}$ Schnyder woods on triangulations with $n$ vertices. By $C_{n}$ we denote the $n$th Catalan 
number $C_{n}=\left(\begin{array}{c}2 n \\ n\end{array}\right) /(n+1)$. Hence, asymptotically there are about $16^{n}$ Schnyder woods on triangulations with $n$ vertices. Tutte's classic result [38] yields that there are asymptotically about $9.48^{n}$ plane triangulations on $n$ vertices. See [27] for a proof of Tutte's formula using Schnyder woods. The two results together imply that a triangulation with $n$ vertices has on average about $1.68^{n}$ Schnyder woods. The next theorem is concerned with the maximum number of Schnyder woods on a fixed triangulation.

Theorem 6 Let $\mathcal{T}_{n}$ denote the set of all plane triangulations with $n$ vertices and $\mathcal{S}(T)$ the set of Schnyder woods of $T \in \mathcal{T}_{n}$. Then,

$$
2.37^{n} \leq \max _{T \in \mathcal{T}_{n}}|\mathcal{S}(T)| \leq 3.56^{n} .
$$

The upper bound follows from Proposition 1 by using that for $d(v) \geq 3$

$$
\left(\begin{array}{c}
d(v) \\
3
\end{array}\right) \cdot 2^{1-d(v)} \leq \frac{5}{8}
$$

For the proof of the lower bound we use the augmented triangular grid $T_{k, \ell}^{*}$. Figure 7 shows a canonical Schnyder wood on $T_{k, \ell}^{*}$ in which the vertical edges are directed up, the horizontal edges to the right and diagonal ones left-down.
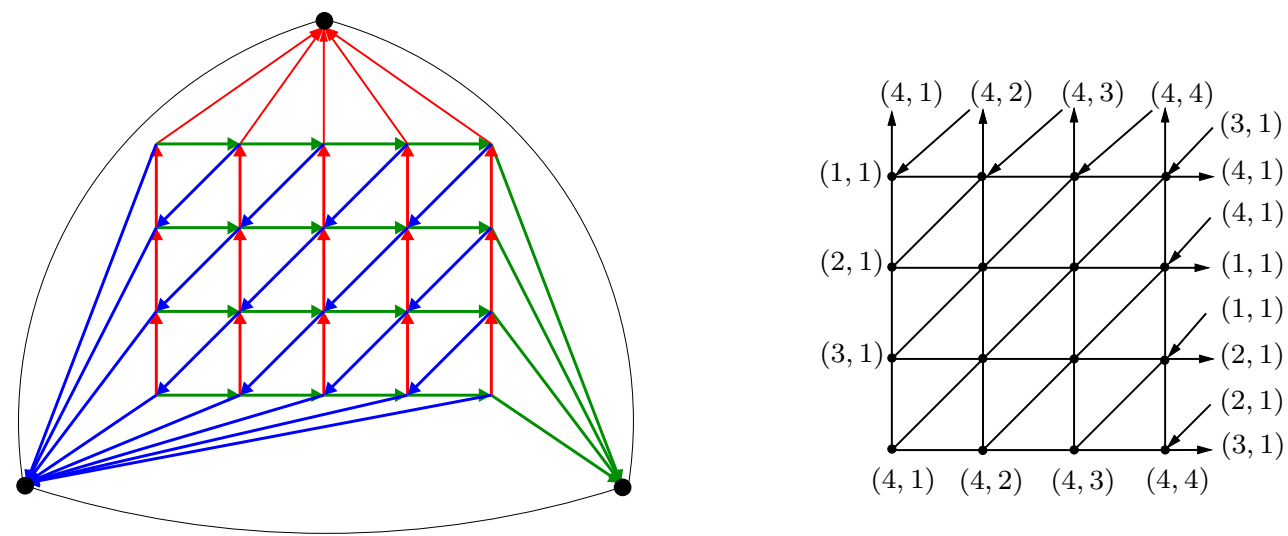

Figure 7: The graphs $T_{4,5}^{*}$ with a canonical Schnyder wood and $T_{4,4}$ with the additional edges simulating Baxter's boundary conditions.

Instead of working with the 3 -orientations of $T_{k, \ell}^{*}$ we use $\alpha^{*}$-orientations of $T_{k, \ell}$ where

$$
\alpha^{*}(i, j)= \begin{cases}3 & \text { if } 2 \leq i \leq k-1 \text { and } 2 \leq i \leq \ell-1 \\ 1 & \text { if }(i, j) \in\{(1,1),(1, \ell),(k, \ell)\} \\ 2 & \text { otherwise }\end{cases}
$$

For the sake of simplicity, we refer to $\alpha^{*}$-orientations of $T_{k, \ell}$ as 3 -orientations.

Intuitively, $T_{k, \ell}$ promises to be a good candidate for a lower bound because the canonical orientation shown in Figure 7 on the left has many directed cycles. We formalize this intuition in the next proposition, which we restrict to the case $k=\ell$ only to make the notation easier. 
Proposition 3 The graph $T_{k, k}^{*}$ has at least $2^{5 / 4(k-1)^{2}}$ Schnyder woods. For $k$ big enough $T_{k, k}^{*}$ has

$$
2.37^{k^{2}+3} \leq\left|\mathcal{S}\left(T_{k, k}^{*}\right)\right| \leq 2.599^{k^{2}+3}
$$

Proof. The face boundaries of the triangles of $T_{k, k}$ can be partitioned into two classes $\mathcal{C}$ and $\mathcal{C}^{\prime}$ of directed cycles, such that each class has cardinality $(k-1)^{2}$ and no two cycles from the same class share an edge. Thus, a cycle $C \in \mathcal{C}^{\prime}$ shares an edge with three cycles from $\mathcal{C}$ if it does not share an edge with the outer face of $T_{k, k}$ and otherwise it shares an edge with one or two cycles from $\mathcal{C}$.

For any subset $D$ of $\mathcal{C}$ reversing all the cycles in $D$ yields a 3-orientation of $T_{k, k}$, and we can encode this orientation as a $0-1$-sequence of length $(k-1)^{2}$. After performing the flips of a given 0-1-sequence $a$, an inner cycle $C^{\prime} \in \mathcal{C}^{\prime}$ is directed if and only if either all or none of the three cycles sharing an edge with $C^{\prime}$ have been reversed. If $C^{\prime} \in \mathcal{C}^{\prime}$ is a boundary cycle, then it is directed if and only none of the adjacent cycles from $\mathcal{C}$ has been reversed. Thus the number of different cycle flip sequences on $\mathcal{C} \cup \mathcal{C}^{\prime}$ is bounded from below by

$$
\sum_{a \in\{0,1\}^{(k-1)^{2}}} 2^{\sum_{C^{\prime} \in \mathcal{C}^{\prime}} X_{C^{\prime}}(a)} .
$$

Here $X_{C^{\prime}}(a)$ is an indicator function which takes value 1 if $C^{\prime}$ is directed after performing the flips of $a$ and 0 otherwise.

We now assume that every $a \in\{0,1\}^{(k-1)^{2}}$ is chosen uniformly at random. The expected value of the above function is then

$$
\mathbb{E}\left[2^{\sum X_{C^{\prime}}}\right]=\frac{1}{2^{(k-1)^{2}}} \sum_{a \in\{0,1\}^{(k-1)^{2}}} 2^{\sum_{C^{\prime} \in \mathcal{C}^{\prime}} X_{C^{\prime}}(a)} .
$$

Jensen's inequality $\mathbb{E}[\varphi(X)] \geq \varphi(\mathbb{E}[X])$ holds for a random variable $X$ and a convex function $\varphi$. Using this we derive that

$$
\mathbb{E}\left[2^{\left.\sum X_{C^{\prime}}\right]} \geq 2^{\mathbb{E}\left[\sum X_{C^{\prime}}\right]}=2^{\sum \mathbb{P}\left[C^{\prime} \text { flippable }\right]} .\right.
$$

The probability that $C^{\prime}$ is flippable is at least $1 / 4$. For $C^{\prime}$ which does not include a boundary edge the probability depends only on the three cycles from $\mathcal{C}$ that share an edge with $C^{\prime}$ and two out of the eight flip vectors for these three cycles make $C^{\prime}$ flippable. A similar reasoning applies for $C^{\prime}$ including a boundary edge. Altogether this yields that

$$
\sum_{a \in\{0,1\}^{(k-1)^{2}}} 2^{\sum_{C^{\prime} \in \mathcal{C}^{\prime}} X_{C^{\prime}}(a)} \geq 2^{(k-1)^{2}} \cdot 2^{(k-1)^{2} / 4} .
$$

Different cycle flip sequences yield different Schnyder woods. The orientation of an edge is easily determined. The edge direction is reversed with respect to the canonical orientation if and only if exactly one of the two cycles on which it lies has been flipped. We can tell a flip sequence apart from its complement by looking at the boundary edges. 
For the upper bound we use Baxter's result for Eulerian orientations on the torus $T_{k, \ell}^{T}$ (see Sections 2.2 and 2.3). Every 3-orientation of $T_{k, \ell}$ plus the wrap-around edges, oriented as shown in Figure 7 on the right, yields a Eulerian orientation of $T_{k, \ell}^{T}$. We deduce that $T_{k, \ell}^{*}$ has at most $2.599^{n}$ Schnyder woods.

Remark. Let us briefly come back to the number of Eulerian orientations of $T_{k, \ell}^{T}$, which was mentioned in Sections 2.2 and 2.3 and in the above proof. There are only $2^{2(k+\ell)-1}$ different orientations of the wrap-around edges. By the pigeon hole principle there is an orientation of these edges which can be extended to a Eulerian orientation of $T_{k, \ell}^{T}$ in asymptotically $(3 \sqrt{3} / 2)^{k \ell}$ ways. Thus, there are out-degree functions $\alpha_{k \ell}$ for $T_{k, \ell}$ such that

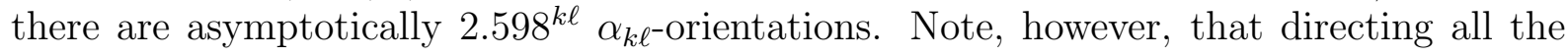
wrap-around edges away from the vertex to which they are attached in Figure 7 induces a unique Eulerian orientation of $T_{k, \ell}$.

We have not been able to specify orientations of the wrap-around edges, which allow to conclude that $T_{k, \ell}$ has $(3 \sqrt{3} / 2)^{k \ell}$ 3-orientations with these boundary conditions. In particular we have no proof that Baxter's result also gives a lower bound for the number of 3-orientations.

\subsection{Schnyder Woods on the Grid and 3-Connected Planar Maps}

In this section we discuss bounds on the number of Schnyder woods on 3-connected planar maps. The lower bound comes from the grid. The upper bound for this case is much larger than the one for triangulations. This is due to the encoding of Schnyder woods by 3 -orientations on the primal dual completion graph, which has more vertices. We summarize the results of this section in the following theorem.

Theorem 7 Let $\mathcal{M}_{n}^{3}$ be the set of 3-connected planar maps with $n$ vertices and $\mathcal{S}(M)$ denote the set of Schnyder woods of $M \in \mathcal{M}_{n}^{3}$. Then,

$$
3.209^{n} \leq \max _{M \in \mathcal{M}_{n}^{3}}|\mathcal{S}(M)| \leq 8^{n}
$$

The example used for the lower bound is the square grid $G_{k, \ell}$.

Theorem 8 For $k, \ell$ big enough the number of Schnyder woods of the augmented grid $G_{k, \ell}^{*}$ is asymptotically $\left|\mathcal{S}\left(G_{k, \ell}^{*}\right)\right| \approx 3.209^{k \ell}$.

Proof. The graph induced by the non-rigid edges in the primal dual completion $\widetilde{G}_{k, \ell}^{*}$ of $G_{k, \ell}^{*}$ is $G_{2 k-1,2 \ell-1}-(2 k-1,1)$. This is a square grid of roughly twice the size as the original and with the lower left corner removed. The rigid edges can be identified using the fact that $\alpha_{S}\left(v_{\infty}\right)=0$ and deleting them induces $\alpha_{S}^{\prime}$ on $G_{2 k-1,2 \ell-1}-(2 k-1,1)$. The new $\alpha_{S}^{\prime}$ only differs from $\alpha_{S}$ for vertices, which are incident to an outgoing rigid edge, and it turns out, that $\alpha_{S}^{\prime}(v)=d(v)-1$ for all primal or dual vertices and $\alpha_{S}^{\prime}(v)=1$ for all edge vertices of $G_{2 k-1,2 \ell-1}-(2 k-1,1)$. Thus, a bijection between $\alpha_{S}^{\prime}$-orientations and perfect matchings of $G_{2 k-1,2 k-\ell}-(2 k-1,1)$ is established by identifying matching edges 
with edges directed away from edge vertices. The closed form expression for the number of perfect matchings of $G_{2 k-1,2 k-\ell}-(2 k-1,1)$ is known (see [21]) to be

$$
\prod_{i=1}^{k} \prod_{j=1}^{\ell}\left(4-2 \cos \frac{\pi i}{k}-2 \cos \frac{\pi j}{\ell}\right) .
$$

The number of perfect matchings of $G_{2 k-1,2 \ell-1}-(2 k-1,1)$ is sandwiched between that of $G_{2 k-2,2 \ell-2}$ and that of $G_{2 k, 2 \ell}$. Therefore the asymptotic behavior is the same and in [24], the limit of the number of perfect matchings of $G_{2 k, 2 \ell}$, denoted as $\Phi(2 k, 2 \ell)$, is calculated to be

$$
\lim _{k, \ell \rightarrow \infty} \frac{\log \Phi(2 k, 2 \ell)}{2 k \cdot 2 \ell}=\frac{\log 2}{2}+\frac{1}{4 \pi^{2}} \int_{0}^{\pi} \int_{0}^{\pi} \log \left(\cos ^{2}(x)+\cos ^{2}(y)\right) d x d y \approx 0.29 .
$$

This implies that $G_{k, \ell}^{*}$ has asymptotically $e^{4 \cdot 0.29 \cdot k \ell} \approx 3.209^{k \ell}$ Schnyder woods.
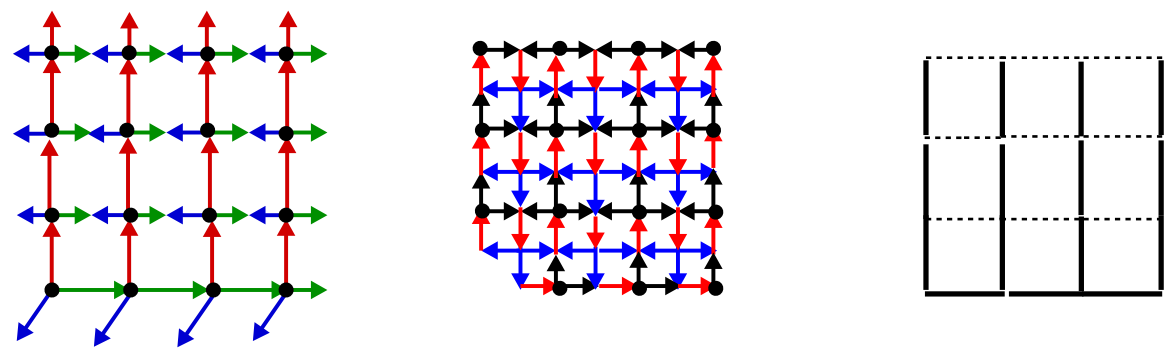

Figure 8: A Schnyder wood on $G_{4,4}^{*}$, the reduced primal dual completion $G_{7,7}-(7,1)$ with the corresponding orientation and the associated spanning tree.

Remark. In [36] Temperley discovered a bijection between spanning trees of $G_{k, \ell}$ and perfect matchings of $G_{2 k-1,2 \ell-1}-(2 k-1,1)$. Thus, Schnyder woods of $G_{k, \ell}^{*}$ are in bijection with spanning trees of $G_{k, \ell}$, see Figure 8. This bijection can be read off directly from the Schnyder wood: the unidirected edges not incident to a special vertex form exactly the related spanning tree. Encoding both, the Schnyder woods and the spanning trees, as $\alpha$-orientations also gives an immediate proof of this bijection.

We now turn to the proof of the upper bound stated in Theorem 7. The proof uses the upper bound for Schnyder woods on plane triangulations, see Theorem 6 . We define a triangulation $T_{M}$ such that there is in injective mapping of the Schnyder woods of $M$ to the Schnyder woods of $T_{M}$. The triangulation $T_{M}$ is obtained from $M$ by adding a vertex $v_{F}$ to every face $F$ of $M$ with $|F| \geq 4$, see Figure 9 . The generic structure of a bounded face of a Schnyder wood is shown on the left in the top row of Figure 9, for a proof see [13]. The three edges, which do not lie on the boundary of the triangle, are the special edges of $F$.

A vertex $v_{F}$ is adjacent to all the vertices of $F$. A Schnyder wood of $M$ can be mapped special edges to a Schnyder wood of $T_{M}$ using the generic structure of the bounded faces as shown in Figure 9. The green-blue non-special edges of $F$ become green unidirected. Their blue 

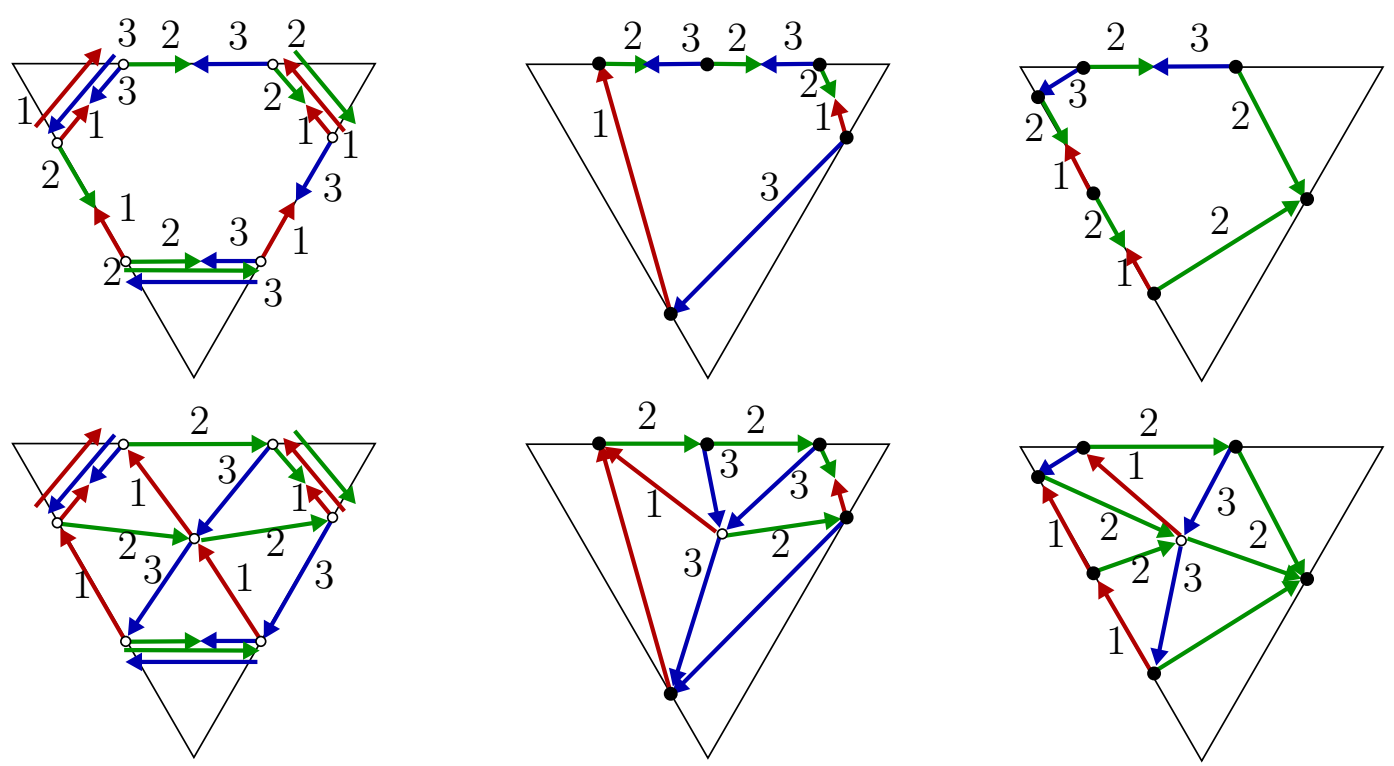

Figure 9: A Schnyder wood on a map $M$ induces a Schnyder wood on $T_{M}$. The three special edges of a face are those, which do not lie on the black triangle.

parts are substituted by unidirected blue edges pointing from their original start-vertex towards $v_{F}$. Similarly the blue-red non-special edges become blue unidirected and the red-green ones red unidirected. Three of the edges incident to $v_{F}$ are still undirected at this point. They are directed away from $v_{F}$ and colored in accordance with (W3).

Let two different Schnyder woods be given that have different directions or colors on an edge $e$. That the map is injective can be verified by comparing the edges on the boundary of the two triangles on which the edge $e$ lies in $T_{M}$.

Thus, it suffices to bound the number of Schnyder woods of $T_{M}$. We do this by specializing Proposition 1. We denote the set of vertices of $T_{M}$ that correspond to faces of size 4 in $M$ by $F_{4}$ and its size by $f_{4}$ and similarly $F_{\geq 5}$ and $f_{\geq 5}$ are defined. Note that $I=F_{4} \cup F_{\geq 5}$ is an independent set and $T_{M}$ has a spanning tree in which all the vertices from $I$ are leaves. Let $n_{T}$ denote the number of vertices of $T_{M}$. Then, $T_{M}$ has at most

$$
2^{3 n_{T}-6-n_{T}} \cdot \prod_{v \in I}\left(\frac{1}{2^{d(v)-1}}\left(\begin{array}{c}
d(v) \\
3
\end{array}\right)\right) \leq 4^{n+f_{4}+f_{\geq 5}} \cdot\left(\frac{1}{2}\right)^{f_{4}} \cdot\left(\frac{5}{8}\right)^{f_{\geq 5}}=4^{n} \cdot 2^{f_{4}} \cdot\left(\frac{5}{2}\right)^{f_{\geq 5}}
$$

Schnyder woods. Note that $n+f_{4}+f_{\geq 5}+f_{4}+2 f_{\geq 5} \leq m+f_{4}+2 f_{\geq 5} \leq 3 n-6$ which implies that $f_{4}+\frac{3}{2} f_{\geq 5} \leq n$. Maximizing equation (4) under this condition yields that the maximum $8^{n}$ is attained when $f_{4}=n$. Thus $M$ has no more than $8^{n}$ Schnyder woods.

The proof of the lower bound $3.209^{n}$ involves the result about the number of perfect matchings of the square grid. This result makes use of non-combinatorial methods. Therefore, we complement this bound with a result for another graph family, which uses a straight-forward analysis, but still yields that these graphs have more Schnyder woods than the triangular grid, see Section 3.1. 
The graph we consider is the filled hexagonal grid $H_{k, \ell}$, see Figure 10. Neglecting boundary effects he hexagonal grid has twice as many vertices as hexagons. This can be seen by associating with every hexagon the vertices of its northwestern edge. Thus, neglecting boundary effects, the filled hexagonal grid has five vertices per hexagon. The boundary effects will not hurt our analysis because $H_{k, \ell}$ has only $2(k+\ell)$ boundary vertices but $5 \cdot k \ell+2(k+l)$ vertices in total.

Proposition 4 For $k, \ell$ big enough the filled hexagonal grid $H_{k, \ell}$ has

$$
2.63^{n} \leq\left|\mathcal{S}\left(H_{k, \ell}\right)\right| \leq 6.07^{n} .
$$
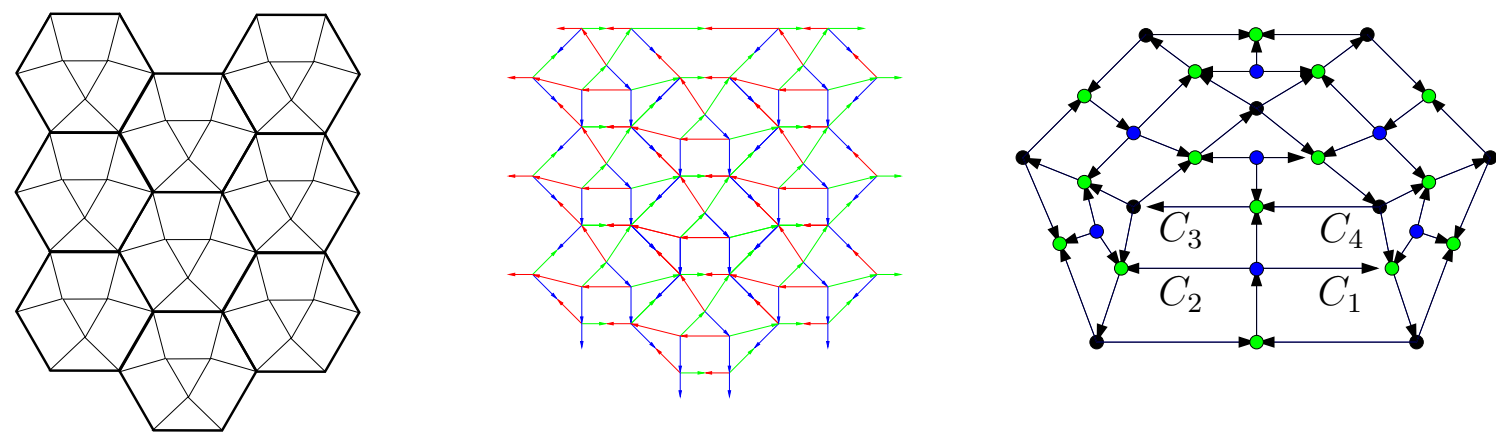

Figure 10: The filled hexagonal grid $H_{3,3}$, a Schnyder wood on this grid and the primaldual suspension of a hexagonal building block of $H_{k, \ell}$. Primal vertices are black, face vertices blue and edge vertices green.

Proof. We count how many different orientations we can have on a filled hexagon. We do this using the bijection from Theorem 4. The right part of Figure 10 shows a feasible $\alpha_{S}$-orientation of a filled hexagon. Note that this orientation is feasible on the boundary when we glue together the filled hexagons to a grid $H_{k, \ell}$ and add a triangle of three special vertices around the grid. We flip only boundary edges of a hexagon which belong to a 4 -face in this hexagon. As these edges belong to a triangle in the hexagon on their other side, the cycle flips in any two filled hexagons can be performed independently.

Let us now count how many orientations a filled hexagon admits, see the right part of Figure 10 for the definition of the cycles $C_{1}, C_{2}, C_{3}$ and $C_{4}$. If the 6-cycle induced by the central triangle is directed as shown in the rightmost part of Figure 10 then we can flip either $C_{1}$ or $C_{2}$ and if $C_{2}$ is flipped, $C_{3}$ can be flipped as well. This yields $4^{3}$ orientations, as the situation is the same at the other two 4-faces of the hexagon. If the 6-cycle is flipped the same calculation can be done with $C_{3}$ replaced by $C_{4}$. This makes a total of $2 \cdot 4^{3}=128$ orientations per filled hexagon. That is, there are at least $128^{k \cdot \ell} \geq 2.639^{5 \cdot k \cdot \ell}$ orientations of $H_{k, \ell}$.

We start the proof of the upper bound by collecting some statistics about $H_{k, \ell}$. As mentioned above, $H_{k, \ell}$ has $n=5 \cdot k \cdot \ell$ interior vertices, $12 \cdot k \ell$ edges and $7 \cdot k \ell$ faces. Thus, the primal-dual completion has $48 \cdot k \ell$ edges. There is no choice for the orientation of the edges incident to the $3 \cdot 4 / 7 \cdot f=12 \cdot k \ell$ face vertices of triangles. We can choose a 
spanning tree $T$ on the remaining $5 \cdot k \ell+12 \cdot k \ell+3 \cdot k \ell$ vertices such that all face vertices are leafs and proceed as in the proof of Proposition 1, but using that we know the number of edges exactly. Since in the independent set of the remaining face vertices all of them have degree 4 and required out-degree 3 , they contribute a factor of $1 / 2$ each. Thus, there are at most $2^{(48-12-20) k \cdot \ell} \cdot 2^{-3 \cdot k \ell}=2^{13 \cdot k \ell} \leq 6.07^{n}$ Schnyder woods on $H_{k, \ell}$.

\section{Counting 2-Orientations}

Felsner et al. [16] present a theory of 2-orientations of plane quadrangulations, which shows many similarities with the theories of Schnyder woods for triangulations. A quadrangulation is a planar map such that all faces have cardinality four. A 2-orientation of a quadrangulation $Q$ is an orientation of the edges such that all vertices but two non-adjacent ones on the outer face have out-degree 2.

In [15] it is shown that 2-orientations on quadrangulations with $n$ inner quadrangles are counted by the Baxter-number $B_{n+1}$. Hence asymptotically there are about $8^{n} 2$ orientations on quadrangulations with $n$ vertices. Tutte gave an explicit formula for rooted quadrangulations. A bijective proof of Tutte's formula is contained in the thesis of Fusy [18]. The formula implies that asymptotically there are about $6.75^{n}$ quadrangulations on $n$ vertices. The two results together yield that a quadrangulation with $n$ vertices has on average about $1.19^{n}$ 2-orientations.

(a)

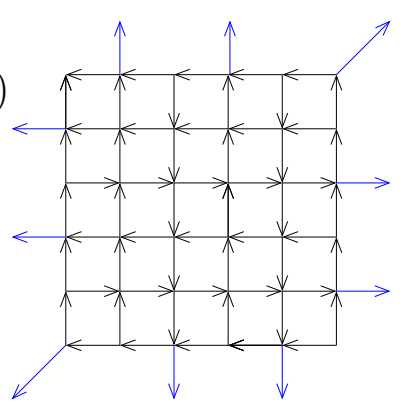

$(\mathrm{b})$

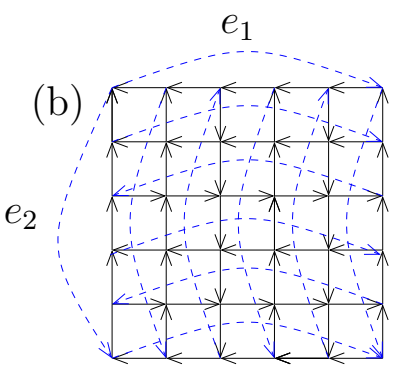

(c)

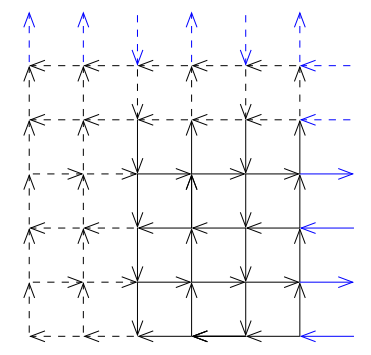

2-

orientation of a quadrangulation

Figure 11: A 2-orientation of $G_{6,6}^{\square}$, the corresponding Eulerian orientation $X$ of $G_{6,6}^{T}$ and an alternating orientation of $G_{4,4}^{T}$ that can be extended to $X$.

We now give a lower bound for the number of 2-orientations of $G_{k, \ell}^{\square}$. The proof method via transfer matrices and eigenvalue estimates comes from Calkin and Wilf [7]. There it is used for asymptotic enumeration of independent sets of the grid graph. Let $\mathcal{Z}(Q)$ denote the set of all 2-orientations of a quadrangulation $Q$, with fixed sinks.

Proposition 5 For $k, \ell$ big enough $G_{k, \ell}^{\square}$ has

$$
1.537^{k \ell} \leq\left|\mathcal{Z}\left(G_{k, \ell}^{\square}\right)\right| \leq(8 \cdot \sqrt{3} / 9)^{k \ell} \leq 1.5397^{k \ell}
$$


Proof. We consider 2-orientations of $G_{k, \ell}^{\square}$ with sinks $(1,1)$ and $v_{\infty}$. These 2-orientations induce Eulerian orientations of $G_{k, \ell}^{T}$. The wrap-around edges inherit the direction of the respective edges incident to $v_{\infty}$ (see Section 2.2) and $e_{1}, e_{2}$ are directed away from $(1,1)$. Therefore $G_{k, \ell}^{\square}$ has at most as many 2-orientations as $G_{k, \ell}^{T}$ has Eulerian orientations, which implies the claimed upper bound.

Conversely a Eulerian orientation of $G_{k, \ell}^{T}$ in which the wrap-around edges have these prescribed orientations induces a 2-orientation of $G_{k, \ell}^{\square}$. Such Eulerian orientations are called almost alternating orientations in the sequel, see Figure 11 (b).

Proving a lower bound for the number of almost alternating Eulerian orientations yields a lower bound for the number of 2-orientations of $G_{k, \ell}^{\square}$.

For the sake of simplicity we will work with alternating orientations of $G_{k-2, \ell-2}^{T}$ instead of almost alternating ones of $G_{k, \ell}^{T}$. In these Eulerian orientations the wrap-around edges are directed alternatingly up and down respectively left and right, see Figure 11 (c). It is easy to see that this is a lower bound for the number of almost alternating orientations of $G_{k, \ell}^{T}$. Since we are interested in an asymptotic lower bound there is no difference in counting alternating orientations of $G_{k-2, \ell-2}^{T}$ and $G_{k, \ell}^{T}$ from our point of view and we will continue working with alternating orientations of $G_{k, \ell}^{T}$ to keep then notation simple.

Consider a vertex column $V_{j}^{C}$ of $G_{k, \ell}^{T}$ and the edge columns $E_{j-1}^{C}$ and $E_{j}^{C}$. Let $X_{1}, X_{2}$ be orientations of $E_{j-1}^{C}$ respectively $E_{j}^{C}$. Let $\delta\left(X_{1}, X_{2}\right)=1$ if and only if the edges induced by $V_{j}^{C}$ can be oriented such that all the vertices of $V_{j}^{C}$ have out-degree 2. Let $\delta_{U}\left(X_{1}, X_{2}\right)=1$ respectively $\delta_{D}\left(X_{1}, X_{2}\right)=1$ if and only if $\delta\left(X_{1}, X_{2}\right)=1$ and the wrap-around edge induced by $V_{j}$ is directed upwards respectively downwards. Note that

$$
\delta_{U}\left(X_{1}, X_{1}\right)=1=\delta_{D}\left(X_{1}, X_{1}\right)
$$

and

$$
\delta_{U}\left(X_{1}, X_{2}\right)=1 \Longleftrightarrow \delta_{D}\left(X_{2}, X_{1}\right)=1 .
$$

We define two transfer matrices $T_{U}(2 k)$ and $T_{D}(2 k)$. These are square 0 -1-matrices with the rows and columns indexed by the $\left(\begin{array}{c}2 k \\ k\end{array}\right)$ orientations of an edge column of size $2 k$, that have $k$ edges directed to the right. The transfer matrices are defined by $\left(T_{U}(2 k)\right)_{X_{1}, X_{2}}$ $=\delta_{U}\left(X_{1}, X_{2}\right)$ and $\left(T_{D}(2 k)\right)_{X_{1}, X_{2}}=\delta_{D}\left(X_{1}, X_{2}\right)$. Hence $T_{U}(2 k)=T_{D}(2 k)^{T}$ and $T_{2 k}=$ $T_{U}(2 k) \cdot T_{D}(2 k)$ is a real symmetric non-negative matrix with positive diagonal entries. From the combinatorial interpretation it can be seen that $T_{2 k}$ is primitive, that is there is an integer $\ell \geq 1$ such that all entries of $T_{2 k}^{\ell}$ are positive and thus the Perron-Frobenius Theorem can be applied. Hence, $T_{2 k}$ has a unique eigenvalue $\Lambda_{2 k}$ with largest absolute value, its eigenspace is 1-dimensional and the corresponding eigenvector is positive.

Let $X_{A}$ be one of the two edge column orientations that have alternating edge directions and $e_{A}$ the vector of dimension $\left(\begin{array}{c}2 k \\ k\end{array}\right)$ that has all entries 0 but the one that stands for $X_{A}$, which is 1 .

The number $c_{A}(2 k, 2 \ell)$ of alternating orientations of $G_{2 k, 2 \ell}^{T}$ is $\left(T_{2 k}^{\ell}\right)_{X_{A}, X_{A}}=\left\langle e_{A}, T_{2 k}^{\ell} e_{A}\right\rangle$. Since the eigenvector belonging to $\Lambda_{k}$ is positive it is not orthogonal to any column of $T_{2 k}$ and we obtain

$$
\lim _{\ell \rightarrow \infty} c_{A}(2 k, 2 \ell)^{1 / \ell}=\lim _{\ell \rightarrow \infty}\left(\left(T_{2 k}^{\ell}\right)_{X_{A}, X_{A}}\right)^{1 / \ell}=\Lambda_{2 k}
$$


where the last equality is justified by an argument known as the power method.

It follows from [22] that the $\operatorname{limit}_{\lim } \rightarrow \infty \Lambda_{2 k}^{1 / k}$ exists, but for the sake of completeness we provide an argument from [7]. We use that $\Lambda_{2 k}^{p} \geq\left\langle v, T_{2 k}^{p} v\right\rangle /\langle v, v\rangle$ for any vector $v$ and that $\left\langle e_{A}, T_{2 k}^{p} e_{A}\right\rangle=\left\langle e_{A}, T_{2 p}^{k} e_{A}\right\rangle$ since both expressions count the number of alternating orientations of $G_{2 k, 2 p}^{T}$.

$$
\left(\Lambda_{2 k}^{1 / k}\right)^{p}=\left(\Lambda_{2 k}^{p}\right)^{1 / k} \geq\left(\left\langle e_{A}, T_{2 k}^{p} e_{A}\right\rangle\right)^{1 / k}=\left(\left\langle e_{A}, T_{2 p}^{k} e_{A}\right\rangle\right)^{1 / k}
$$

Taking limits with respect to $k$ on both sides yields

$$
\left(\liminf _{k \rightarrow \infty} \Lambda_{2 k}^{1 / k}\right)^{p} \geq \liminf _{k \rightarrow \infty}\left(\left\langle e_{A}, T_{2 p}^{k} e_{A}\right\rangle\right)^{1 / k}=\Lambda_{2 p}
$$

which implies $\liminf _{k \rightarrow \infty} \Lambda_{2 k}^{1 / k} \geq \limsup _{p \rightarrow \infty} \Lambda_{2 p}^{1 / p}$. It follows that $\lim _{k \rightarrow \infty} \Lambda_{2 k}^{1 / k}$ exists. Similar arguments as above yield the following.

$$
\Lambda_{2 k}^{p} \geq \frac{\left\langle e_{A} T_{2 k}^{q}, T_{2 k}^{p} T_{2 k}^{q} e_{A}\right\rangle}{\left\langle T_{2 k}^{q} e_{A}, T_{2 k}^{q} e_{A}\right\rangle}=\frac{\left\langle e_{A}, T_{2 k}^{p+2 q} e_{A}\right\rangle}{\left\langle e_{A}, T_{2 k}^{2 q} e_{A}\right\rangle}=\frac{\left\langle e_{A}, T_{2 p+4 q}^{k} e_{A}\right\rangle}{\left\langle e_{A}, T_{4 q}^{k} e_{A}\right\rangle} .
$$

Taking limits with respect to $k$ on both sides yields

$$
\lim _{k \rightarrow \infty} \Lambda_{2 k}^{1 / k} \geq\left(\frac{\Lambda_{4 q+2 p}}{\Lambda_{4 q}}\right)^{1 / p}
$$

We are interested in $\lim _{k \rightarrow \infty} \lim _{\ell \rightarrow \infty} c_{A}(2 k, 2 \ell)^{1 / 4 k \ell}=\lim _{k \rightarrow \infty} \Lambda_{2 k}^{1 / 4 k}$ since $4 k \ell$ is the number of vertices of $G_{2 k, 2 \ell}^{T}$. Using a Mathematica program we have computed $\Lambda_{10}$ and $\Lambda_{8}$ with the result that

$$
\left(\frac{\Lambda_{10}}{\Lambda_{8}}\right)^{1 / 4} \geq\left(\frac{2335.8714}{418.2717}\right)^{1 / 4} \geq 1.537
$$

Remark. We return to the correspondence between 2-orientations of $G_{k, \ell}^{\square}$ and Eulerian orientations of $G_{k, \ell}^{T}$, that was mentioned at the beginning of the last proof. By the pigeon hole principle, there must be a sequence of orientations $X_{k, \ell}$ of the wrap-around edges that extends asymptotically to $(8 \cdot \sqrt{3} / 9)^{k \ell}$ Eulerian orientations of $G_{k, \ell}^{T}$. This implies that for $k, \ell$ big enough there is an $\alpha_{k, \ell}$ on $G_{k, \ell}$ such that there are $(8 \cdot \sqrt{3} / 9)^{k \ell} \alpha_{k, \ell^{-}}$orientations of $G_{k, \ell}$. This $\alpha_{k, \ell}$ satisfies $\alpha_{k \ell}(v)=2$ for every inner vertex $v$ and $\alpha_{k \ell}(w) \in\{0,1,2\}$ for every boundary vertex $w$. We call $\alpha$-orientations of this type inner 2-orientations of the grid.

We think that $G_{k, \ell}^{\square}$ has asymptotically $(8 \cdot \sqrt{3} / 9)^{k \ell}$ 2-orientations. But we were not able to show this, just like for the case of the triangular grid, see the last remark of Section 3.1. 
Theorem 9 Let $\mathcal{Q}_{n}$ denote the set of all plane quadrangulations with $n$ vertices and $\mathcal{Z}(Q)$ the set of 2-orientations of $Q \in \mathcal{Q}_{n}$. Then, for $n$ big enough

$$
1.53^{n} \leq \max _{Q \in \mathcal{Q}_{n}}|\mathcal{Z}(Q)| \leq 1.91^{n}
$$

Proof. The lower bound is that from Proposition 5. An upper bound of $2^{n}$ follows immediately from Lemma 1. Note that we may assume that $Q$ does not have vertices of degree 2 , because their incident edges would be rigid. Bonsma $[6,5]$ shows that trianglefree graphs of minimal degree 3 have a spanning tree $T$ with more than $n / 3$ leafs. As $Q$ is bipartite, $T$ has as a set $I$ of at least $n / 6$ leafs which is an independent set of $Q$. As in Proposition 1 , this yields that there are at most $2^{n} \cdot(3 / 4)^{n / 6} \leq 1.91^{n} 2$-orientations of $Q$.

\section{Counting Bipolar Orientations}

We first give an overview of the definitions and facts about bipolar orientations that we need in this section. A good starting point for further reading about bipolar orientations is [11].

Let $G$ be a connected graph and $e=s t$ a distinguished edge of $G$. An orientation $X$ of the edges of $G$ is an e-bipolar orientation of $G$ if it is acyclic, $s$ is the only vertex without incoming edges and $t$ is the only vertex without outgoing edges. We call $s$ and $t$ the source respectively sink of $X$. There are many equivalent definitions of bipolar e-bipolar orientaorientations, c.f. [11].

Figure 5.2 shows examples of plane bipolar orientations. Note that in this case it is enough to have vertices $s, t$ on the outer face, they need not be adjacent. The following characterization of plane bipolar orientations will be useful to keep some proofs in the sequel simple.

Proposition 6 An orientation $X$ of a planar map $M$ with two special vertices $s$ and $t$ on the outer face is a bipolar orientation if and only if it has the following two properties.

(1) Every vertex other than the source $s$ and the sink $t$, has incoming as well as outgoing edges.

(2) There is no directed facial cycle.

Furthermore, the following stronger versions of the above properties hold for every bipolar orientation.

(1') At every vertex other than the source and the sink, the incoming and outgoing edges form two non-empty bundles of consecutive edges.

(2') The boundary of every face has exactly one sink and one source, i.e. consists of two directed paths. 
We omit the proof that properties (1) and (2) imply that $X$ is a bipolar orientation. The proof that every bipolar orientation has properties $\left(1^{\prime}\right)$ and $\left(2^{\prime}\right)$ (and thus properties (1) and (2) as well) can be found in [40] or [35].

The fact that properties $\left(1^{\prime}\right)$ and $\left(2^{\prime}\right)$ characterize bipolar orientations of planar maps yields a bijection between bipolar orientations of a map $M$ and 2-orientations of the angular map, i.e., of the map $\widehat{M}$ on the vertex set $V \cup \mathcal{F}$, where where $\mathcal{F}$ is the set of faces of $M$, and edges $\{v, f\}$ for all incident pairs with $v \in V$ and $F \in \mathcal{F}$. This bijection was first described by Rosenstiehl [30].
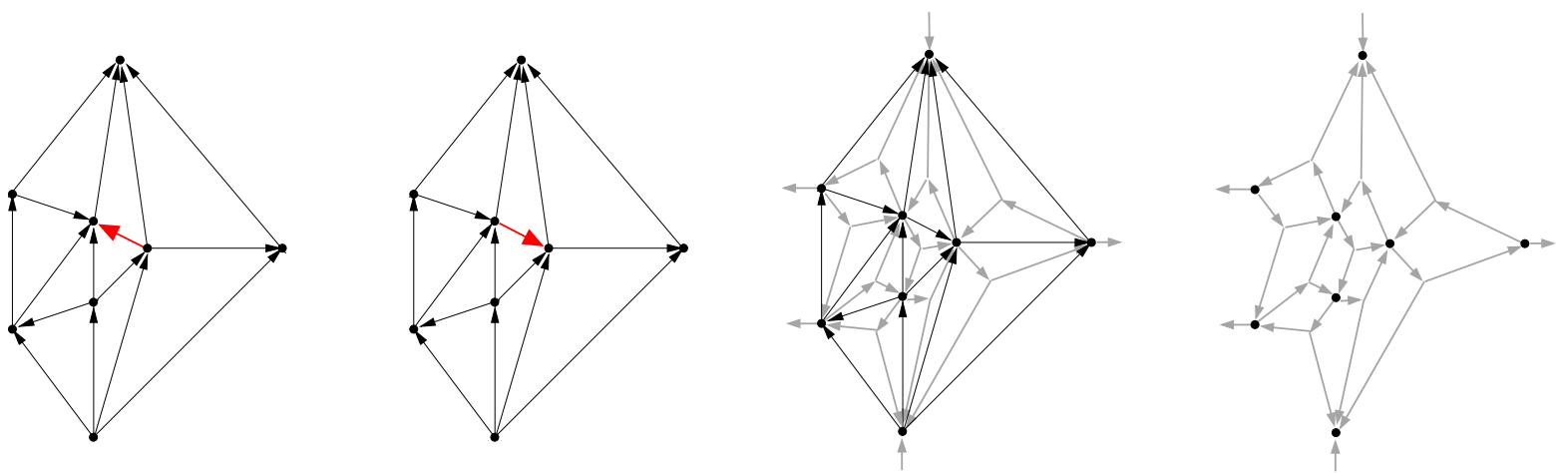

Figure 12: Two bipolar orientations of the same graph with different out-degree sequences and the corresponding $\alpha$-orientation of the angle graph (vertex for the unbounded face omitted).

Since bipolar orientations and 2-orientations of quadrangulations are in bijection, we explain now how the results from Theorems 9 and 11 are related. A triangulation with $n$ vertices has an angle graph with roughly $3 n$ vertices. Hence the upper bound of $1.91^{3 n}$, that Theorem 9 yields for the number of bipolar orientation, is worse then the upper bound from Theorem 11. Conversely, every quadrangulation $Q$ with $n$ vertices is the angle graph of the map obtained by connecting two vertices of one of its partition classes by an edge, if they lie on a common 4 -face of $Q$. This might yield a multi-graph if $Q$ has degree 2 vertices. But we may neglect this, since parallel edges must have the same direction in every bipolar orientation. One of the partition classes of $Q$ has size at most $n / 2$, and thus the upper bound from Theorem 11 yields that $Q$ has at most $3.97^{n / 2} 2$ orientations, which is worse than the bound from Theorem 9. The example of the $G_{k, \ell}^{\square}$, which has $1.53^{n}$ 2-orientations is the angle graph of a graph which has roughly $n / 2=: n^{\prime}$ vertices. Therefore this yields only an example with $1.53^{2 n^{\prime}}$ bipolar orientations, which is far away from the bound given in Theorem 11. Conversely, the triangular grid $T_{k, \ell}$, which has at least $2.91^{n}$ bipolar orientations has an angle graph with roughly $3 n=n^{\prime}$ vertices. This yields a quadrangulation with $2.91^{n^{\prime} / 3}$ 2-orientations, which is worse than the bound for the number of 2-orientations that we obtained in Proposition 5. 


\subsection{Counting Bipolar Orientations on the Grid}

We now turn to analyzing the number of bipolar orientations of $G_{k, \ell}$, with source $(1,1)$ and sink $(k, \ell)$ if $k$ is odd and sink $(k, 1)$ if $k$ is even. For the proof of the Theorem, we need sparse sequences. A sparse sequence is a 0 -1-sequences without consecutive 1 s and it is well known that there are $F_{n+2}$ such sequences of length $n$, where $F_{n+2}$ denotes the $(n+2)$ th Fibonacci number.

Theorem 10 Let and $\mathcal{B}\left(G_{k, \ell}\right)$ denote the set of bipolar orientations of $G_{k, \ell}$. For $k, \ell$ big enough the number of bipolar orientations of the grid $G_{k, \ell}$ is bounded by

$$
2.18^{k \ell} \leq\left|\mathcal{B}\left(G_{k, \ell}\right)\right| \leq 2.619^{k \ell} .
$$

Proof. We first prove the lower bound with an argument using directed cycles in a canonical orientation, as in Proposition 3. To make this tool applicable we consider 2orientations of the angle graph $\hat{G}_{k, \ell}$ of $G_{k, \ell}$. Figure 13 shows the angle graph $\hat{G}_{4,5}$. The graph $\hat{G}_{k, \ell}$ has $2 k \ell-3(k+\ell)+4$ squares. All dotted edges are rigid, just like the four edges which are adjacent to a degree 2 vertex. Therefore, we may neglect all these edges in the rest of the proof.

The independent set $I$ of directed cycles in the canonical orientation is marked by dots and includes approximately half of all squares. The set $I^{\prime}$ consists of all squares that are not in $I$. Members of $I^{\prime}$ can be flipped if either the two cycles of $I$ above it, or the two cycles of $I$ below it are flipped, that is in 2 out of 16 cases ( 1 out of 4 for boundary squares). Roughly half of all squares are in $I^{\prime}$.

(a)

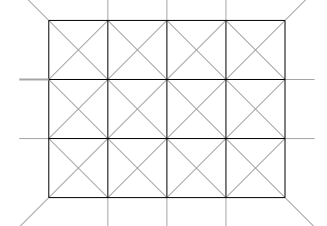

(b)

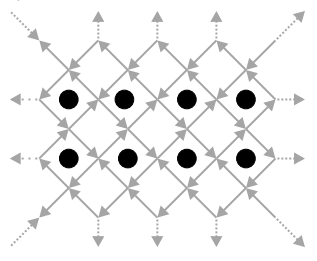

(c)

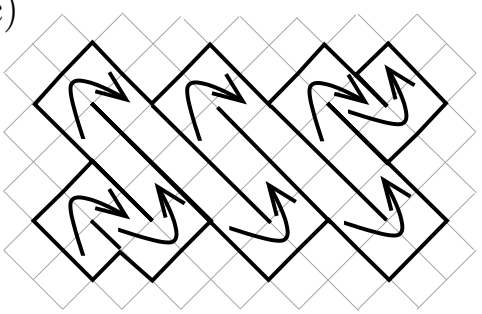

Figure 13: The grid $G_{4,5}$ is shown with its angle graph in blue; a canonical 2-orientation on $\hat{G}_{4,5}$ where red edges all connect to an additional vertex $v_{\infty}$ and the dots mark an independent set of directed cycles; the central part of $\hat{G}_{6,9}$ and the traversal used in the proof of the upper bound.

Thus, there are at least $2^{|I|+\left|I^{\prime}\right| / 8}$ bipolar orientations of $G_{k, \ell}$, which leads to an asymptotic lower bound of $2^{9 k \ell / 8} \approx 2.18^{k \ell}$.

For the proof of the upper bound we use a bijection discovered by Lieb [22]. The bijection relates face 3-colorings where no two squares sharing an edge have the same color and inner 2-orientations of the square grid as shown in Figure 14 (a). Figure 14 (b) shows the face 3-coloring corresponding to the canonical 2-orientation, that we used for the proof of the lower bound. 
(a)

$$
\begin{array}{ll}
R \downarrow G & R \uparrow B \\
B \nmid R & B \nmid G \\
G^{A} R & G \downarrow B
\end{array}
$$

(b)

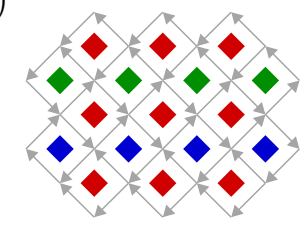

Figure 14: Lieb's bijection between inner 2-orientations and face 3-colorings on the grid.

Here we use this relation on $\hat{G}_{k, \ell}$. We prove an upper bound for the number of face 3-colorings of $\hat{G}_{k, \ell}$. The right part of Figure 13 shows the central part of $\hat{G}_{k, \ell}$ bounded by a thick polygon. We will encode the 3 -coloring on the faces of the central part of $\hat{G}_{k, \ell}$ as a sparse sequence $a$, where $a_{i}$ represents the $i$ th square on the path $P$ indicated by the arrows in the figure.

The set $\mathcal{D}$ of faces, which are not in the central part, has less than $3^{|\mathcal{D}|} 3$-colorings. In the encoding described next, the code for the $i$ th face of the path $P$ depends only on faces in $\mathcal{D}$ and faces of $P$ with index smaller than $i$. Figure 15 shows how the color of the highlighted face is encoded by a 0 or a 1 . The arrows indicate the direction in which we traverse the central part of the graph. There are three cases, one for a face where the path makes no turn and two for the two different types of turn faces. The variables $X, Y, Z$ represent an arbitrary permutation of $R, G, B$.

As for the decoding, it is clear from the figure that the faces marked with an $X$ or $Y$ plus the 0-1 encoding uniquely determine the color of the face in question. Thus the encoding is injective. It remains to show that there cannot be consecutive $1 \mathrm{~s}$ in this sequence. This follows from the observation, that writing a 1 means, that the two faces that will be used for the encoding of the next face on the path have different colors. Thus, this face will be encoded by a 0 .
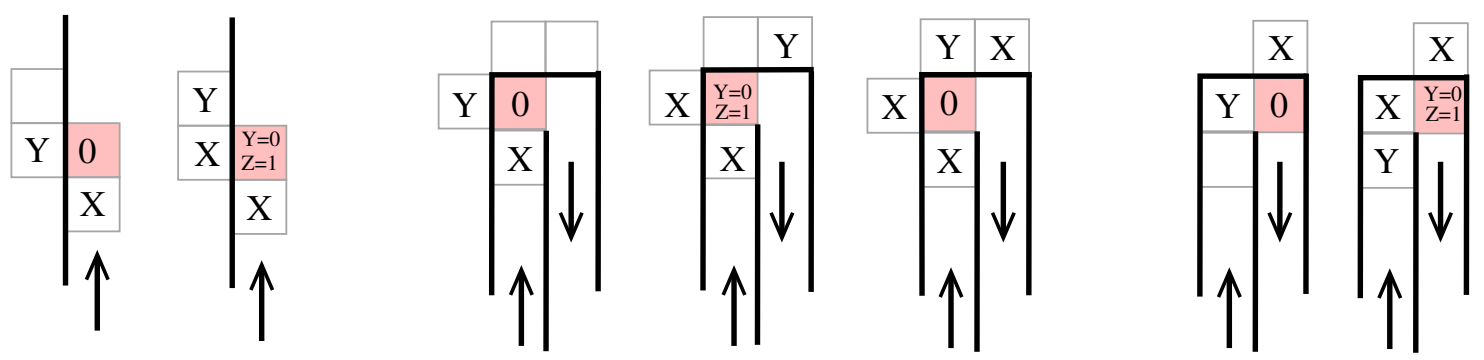

Figure 15: Encoding a 3-coloring by a sparse $0-1$-sequence. On the left the encoding for a square where the path makes no turn, in the center and right for the two different kind of turn faces.

We bound the number of such encodings from above. The set $\mathcal{D}$ can be covered by at most four horizontal plus four vertical rows of faces, thus $|\mathcal{D}| \leq 4(k+\ell)$. The length of the path is bounded by the number of bounded faces of $\hat{G}_{k, \ell}$, which is less than $2 k \ell$. Therefore, there are at most

$$
3^{4(k+\ell)} \cdot F_{2 k \ell+2}
$$


such encodings. Using the asymptotics for the Fibonacci numbers this implies, that there are less than $2.619^{k \ell}$ such encodings for $k, \ell$ big enough.

Lieb's analysis of the number of Eulerian orientations of $T_{k \ell}$ is of interest in this case as well. It allows to improve the upper bound for grids with side lengths ratio one to two.

Proposition 7 For $k$ big enough the number of bipolar orientations of the grid $G_{k, 2 k}$ is bounded by

$$
2.18^{2 k^{2}} \leq\left|\mathcal{B}\left(G_{k, 2 k}\right)\right| \leq 2.38^{2 k^{2}}
$$
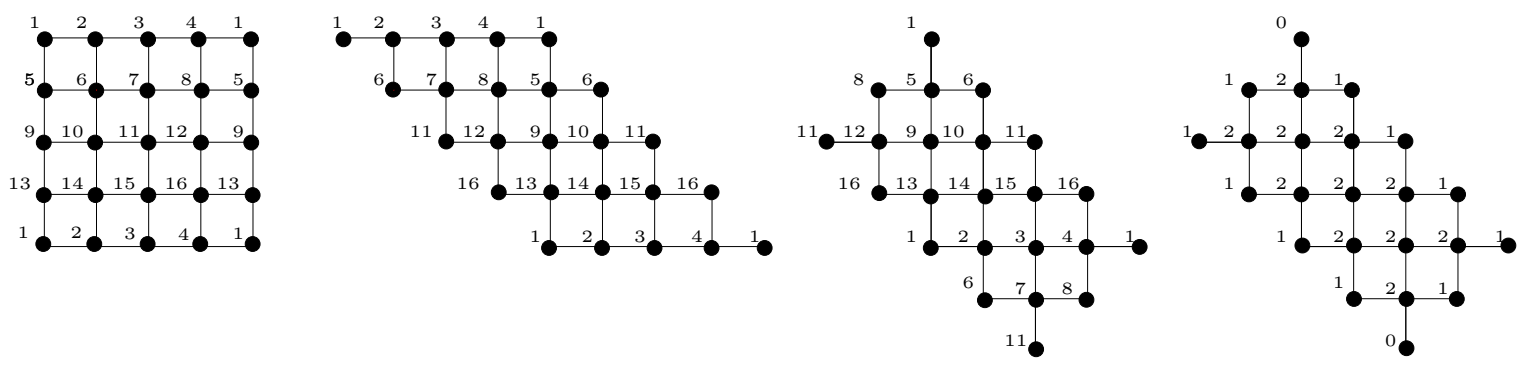

Figure 16: How to obtain the tilted grid $\hat{G}_{5,3}$ from $G_{4,4}^{T}$ with two cuts. The numbers in the first three drawings are the vertex labels, in the last one they indicate $\hat{\alpha}$.

Proof. By $\hat{G}_{k, \ell}^{\prime}$ we denote the graph obtained from $\hat{G}_{k, \ell}$ by deleting $v_{\infty}$ and all incident edges, which are shown as dotted edges in Figure 13. Figure 16 shows how to cut $G_{4,4}^{T}$ in two steps such that the grid looks like $\hat{G}_{3,5}^{\prime}$ (if we do not identify vertices). The last drawing shows, that every $\hat{\alpha}$-orientation of $\hat{G}_{3,5}^{\prime}$ yields a Eulerian orientation of $G_{4,4}^{T}$ when we do the appropriate identifications. In general, this approach yields an injection from the bipolar orientations of $G_{k+1,2 k+1}$ to the Eulerian orientations of $G_{2 k, 2 k}^{T}$. As Lieb [22] has shown that $G_{2 k, 2 k}^{T}$ has asymptotically $(8 \cdot \sqrt{3} / 9)^{4 k^{2}}$ Eulerian orientations, this yields an upper bound of $(64 / 27)^{2 k^{2}}$ for the number of Eulerian orientations of $G_{k+1,2 k+1}$. Every bipolar orientation of $G_{k, 2 k}$ can be complemented to a bipolar orientation of $G_{k+1,2 k+1}$, thus $G_{k, 2 k}$ has at most as many bipolar orientation as $G_{k+1,2 k+1}$. The lower bound follows from the more general claim of Theorem 10.

Remark. The same problems as described in the closing remarks of Sections 3.1 and 4 arise here when trying to show that $G_{k, 2 k}$ actually has $(64 / 27)^{2 k^{2}}$ bipolar orientations by using Lieb's result for the torus.

\subsection{Counting Bipolar Orientations On Planar Maps}

Note that adding edges to the faces of size at least 4 of a planar map $M$ can only increase the number of bipolar orientations by Proposition 6 . Thus we can restrict our considerations to plane inner triangulations in this section. 
Theorem 11 Let $\mathcal{M}_{n}$ denote the set of all planar maps with $n$ vertices and $\mathcal{B}(M)$ the set of all bipolar orientations of $M \in \mathcal{M}_{n}$. Then, for $n$ big enough

$$
2.91^{n} \leq \max _{M \in \mathcal{M}_{n}}|\mathcal{B}(M)| \leq 3.97^{n} .
$$

For the proof we need a couple of facts about Fibonacci numbers, which are summarized in the following lemmas. The Fibonacci numbers are the integer series defined by the recursion

$$
F_{1}=1, F_{2}=1, F_{n}=F_{n-1}+F_{n-2} \text { for } n \geq 3 .
$$

Define $F_{0}=0$ and let $\phi=\frac{1+\sqrt{5}}{2}$ be the Golden Ratio.

Lemma 3 The Fibonacci numbers have the following properties.

- $F_{n}=\frac{\phi^{n}-(1-\phi)^{n}}{\sqrt{5}}$

- $\lim _{n \rightarrow \infty} F_{n}=\frac{\phi^{n}}{\sqrt{5}}$

- $\sum_{i=0}^{n} F_{i} F_{n-i}=\frac{1}{5}\left(n\left(F_{n+1}+F_{n-1}\right)-F_{n}\right)$

The first two are standard results from the vast theory of Fibonacci numbers. The last formula is attributed to Shiwalkar and Deshpande in [34, A001629]. The next lemma summarizes facts about sparse sequences.

Lemma 4 The number of sparse sequences of length $n$ is $F_{n+2}$. Let $r_{n}(i)$ be the number of sparse sequences of length $n$ whose ith entry is 1 . Then,

- $r_{n}(i)=F_{i} \cdot F_{n+1-i}$

- $\sum_{i=1}^{n} r_{n}(i)=\frac{1}{5}\left(2(n+1) F_{n}+n F_{n+1}\right)$

- $\lim _{n \rightarrow \infty} \frac{\sum_{i=1}^{n} r_{n}(i)}{n F_{n+2}}=\frac{1}{\sqrt{5} \phi} \approx 0.2764$

The first identity follows from a construction of sparse sequences of length $n$ from sparse sequences of length $n-1$ plus the string " 0 " and sparse sequences of length $n-2$ plus "01". The second and third identity then follow using the facts from Lemma 3.

Before proving the Theorem 11 we give two results for the number of bipolar orientations of special classes of planar maps.

Proposition 8 A stacked triangulation with $n$ vertices has $2^{n-3}$ bipolar orientations.

Proof. The $K_{4}$ has two bipolar orientations for fixed source and sink. We proceed by induction and assume, that a stacked triangulation with $n$ vertices has $2^{n-3}$ bipolar orientations. Now let $T$ be a stacked triangulation with $n+1$ vertices and $v$ a vertex of degree 3 in $T$. Then, $T-v$ has $2^{n-3}$ bipolar orientations by induction. Now stacking 
$v$ into $T$ again, there are exactly two ways to complete a given bipolar orientation on $T-v$ without violating Properties (1) or (2) from Proposition 6 . Thus, there are $2^{(n+1)-3}$ bipolar orientations of $T$.

Proposition 9 Let $\mathcal{O}_{n}$ be the set of all outerplanar maps with $n$ vertices. Then,

$$
\max _{M \in \mathcal{O}_{n}}|\mathcal{B}(M)|=F_{n-1} \approx 1.618^{n-1} .
$$

Proof. We show first that there are indeed outerplanar maps with $F_{n-1}$ bipolar orientations. Let $T:=T_{2, \ell}$ be the triangular grid with two rows. We consider bipolar orientations of $T$ with source $(1,1)$ and sink $(2, \ell)$. In every such bipolar orientation the boundary edges form two directed paths from $(1,1)$ to $(2, \ell)$. We start by defining the standard bipolar orientation $B_{0}$ of $G^{*}$, which is shown in Figure 17.

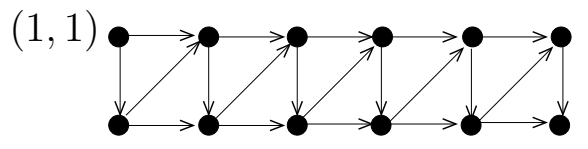

Figure 17: The standard bipolar orientation on $T_{2, \ell}$.

In $B_{0}$ the vertical inner edges are directed downwards and the diagonal ones upwards. Now we encode any other orientation of the inner edges by a sequence $\left(a_{i}\right)_{i=1 \ldots n^{\prime}}$ of length $n^{\prime}=n-3$, where $a_{i}=1$ if the corresponding edge has the opposite direction as in $B_{0}$ and $a_{i}=0$ otherwise. The entries come in the natural left to right order in $\left(a_{i}\right)_{i=1 \ldots n^{\prime}}$. We show that all sparse sequences of length $n^{\prime}$ produce bipolar orientations. In a sparse sequence there are no consecutive $1 \mathrm{~s}$, thus out of the two inner edges incident to a vertex at most one is reversed with respect to $B_{0}$. This guarantees that there is no directed facial 3-cycle. As all vertices have an incoming and an outgoing outer edge the resulting orientation is bipolar, according to Proposition 6.

It remains to show that $F_{n-1}$ is an upper bound for the number of bipolar orientations of any outerplanar map $M$ with $n$ vertices. We may assume that $M$ is a plane inner triangulation. The proof uses induction on the number of vertices and the claim is trivial for $n=3$. Now, let $M$ have $n+1$ vertices and let $s$ be the source vertex. If $M$ has a vertex $x \neq s, t$ of degree 2 with neighbors $v, w$ then the direction of the edge $\{v, w\}$ determines the directions of the edges $\{x, v\}$ and $\{x, w\}$. Therefore, $M$ has at most as many bipolar orientations as $M-x$, that is at most $F_{n-1}$ many. If all vertices but $s$ and $t$ have degree at least three, then $s$ and $t$ have degree 2 and the vertices of every inner edge of $M$ are separated by $s$ and $t$ on the outer cycle. This is because the interior of the boundary cycle on $n+1$ vertices is partitioned into $n-1$ triangles, and thus two of these triangles must share two edges with the boundary, which yields two degree 2 vertices.

So $s$ is incident to only two vertices $v$ and $w$, and we may assume that $v$ has degree 3 in $M$, that is the inner edge $e=\{v, w\}$ is the only inner edge incident to $v$. Now, let $X$ be some bipolar orientation of $M$ in which $e$ is directed from $v$ to $w$. Then, the orientation of $M-s$ induced by $X$ is a bipolar orientation with source $v$. For a bipolar 
orientation $Y$ in which $e$ is oriented from $w$ to $v$, the orientation of $M-s$ induced by $X$ is a bipolar orientation with source $w$ and $v$ is a vertex of degree 2 in $M-s$. This mapping is injective, and thus $M$ has at most as many bipolar orientations as $M-s$ and $M-\{s, v\}$ together, that is $F_{n-1}+F_{n-2}=F_{n}$.

Remark. From the above proof it also follows, that $T_{2, \ell}$ is the only outerplanar map on $2 \ell$ vertices, which has $F_{2 \ell-1}$ bipolar orientations.

The example that gives the lower bound for the number of bipolar orientations of planar maps is the triangular grid $T_{k, k}$ with source $(1,1)$ and sink $(k, k)$.

Proposition 10 Let $T_{k, k}$ be the triangular grid and $k$ big enough. Then,

$$
\left|B\left(T_{k, k}\right)\right| \geq 2.91^{n} .
$$

Proof. We first claim that $T_{k, k}$ has at least $2.618^{k^{2}}$ bipolar orientations. To see this we glue together $k-1$ copies of $T_{2, k}$. Every orientation of $T_{k, k}$ obtained in this way corresponds to a concatenation of $k-1$ sparse sequences of length $2 k-3$, which we call an almost sparse sequence. We denote the set of all such sequences of length $2 k^{2}-5 k+3$ by $S$, the cardinality of $S$ is $F_{2 k-1}^{k-1}$ which is bounded below by $F_{2 k^{2}-5 k+3} \geq 2.618^{k^{2}}$ for $k$ big enough. That each $s \in S$ corresponds to a bipolar orientation of $T_{k, k}$ can be checked using Proposition 6.

The horizontal edge $e_{i, j}:=(i, j) \rightarrow(i, j+1)$ lies on the boundary of two triangles for $2 \leq i \leq k-1$. The other four edges of these triangles are

$$
\{(i, j),(i-1, j+1)\},\{(i, j+1),(i-1, j+1)\},\{(i, j),(i+1, j)\},\{(i, j+1),(i+1, j)\} .
$$

The crucial observation for improving the above bound is, that we can reorient $e_{i, j}$ if and only if the entries belonging to these four edges show one of the two patterns $10 \ldots 01$ or $01 \ldots 10$.

We now choose $k-1$ sparse sequences of length $2 k-3$ independently uniformly at random and concatenate them to obtain a random almost sparse sequence $s \in S$. It follows from the first identity from Lemma 4 with $n=2 k-3$ and $i=2 j-1$ that for $\{(i, j),(i, j+1)\}$ there are $F_{2 j-1} F_{2 k-2 j-1} F_{2 k-1}^{k-2}$ sequences that have $\{(i, j),(i-1, j+1)\}$ marked 1 out of the total $F_{2 k-1}^{k-1}$ sequences. This is used to calculate the probability that the entry for $\{(i, j),(i-1, j+1)\}$ is 1 as

$$
\lim _{k \rightarrow \infty} \frac{F_{2 j-1} F_{2 k-2 j-1} F_{2 k-1}^{k-2}}{F_{2 k-1}^{k-1}}=\lim _{k \rightarrow \infty} \frac{1}{\sqrt{5}} \cdot \phi^{2 j-1} \cdot \phi^{2 k-2 j-1} \cdot \phi^{-2 k+1}=\frac{1}{\sqrt{5} \phi} .
$$

Taking the limit is only justifiable if $(2 j-1) \rightarrow \infty$ and $(2 k-2 j-1) \rightarrow \infty$ for $k \rightarrow \infty$. Therefore we introduce $\delta>0$ and denote the set of horizontal edges with $\delta(k-1) \leq j \leq$ $(1-\delta)(k-1)$ and $2 \leq i \leq k-1$ by $E_{\delta}$. Taking the limit in equation (5) is justified for all $e_{i, j} \in E_{\delta}$. The size of this set is $\left|E_{\delta}\right|=(1-2 \delta)(k-1)^{2}$.

This works analogously for the edge $\{(i, j+1),(i+1, j)\}$ and the events are independent. The pattern $01 \ldots 10$ has the same probability in the limit and the patterns 
mutually exclude each other. Thus, for every $\epsilon>0$ the probability that the edge $\{(i, j),(i, j+1)\} \in E_{\delta}$ can be flipped is

$$
\mathbb{P}\left[\mathbf{1}_{i, j}(s)=1\right] \geq \frac{2}{5 \phi^{2}}-\epsilon
$$

for $k$ big enough.

The rest of the proof is to analyze how many of the flip-patterns we expect for a sparse sequences $s$. Let $Q(s)=\sum_{i, j} \mathbf{1}_{i, j}(s)$ be a random variable counting the number of flippable edges in $s$. We use Jensen's inequality to estimate the number of orientations, which is at least

$$
|S| \cdot \mathbb{E}_{s \in \mathcal{S}}\left[2^{Q(s)}\right] \geq F_{2 k^{2}-5 k+3} \cdot 2^{\mathbb{E}_{s \in \mathcal{S}}[Q(s)]} \geq 2.618^{k^{2}} \cdot 2^{(1-2 \delta)(k-1)^{2}\left(\frac{2}{5 \phi^{2}}-\epsilon\right)} \geq 2.91^{k^{2}}
$$

for $k$ big enough.

Remark. The third identity from Lemma 4 gives the expected number of 1 s that a random entry of a random sparse sequence of length $n$ has. In equation (5) we calculate the expected number of $1 \mathrm{~s}$ in a random sequence of length $n$ at a fixed entry which is far enough away from the boundary. The fact that these two values agree shows that the expected number of $1 \mathrm{~s}$ at a fixed entry of a sparse sequence does not depend strongly on the choice of the entry.

The following relation is useful to upper bound the number of bipolar orientations for plane inner triangulations. It has been presented with a different proof in [25]. Let $\mathcal{F}_{b}$ be the set of bounded faces of $M$ and $\mathcal{B}$ the set of bipolar orientations of $M$. Fix a bipolar orientation $B$. The boundary of every triangle $\Delta \in \mathcal{F}_{b}$ consists of a path of length two and a direct edge from the source to the sink of $\Delta$. We say that $\Delta$ is a + triangle of $B$ if looking along the direct source-sink edge the triangle is on the left. Otherwise, if the triangle is on the right of the edge we speak of a - triangle, see Figure 18. We use this notation to define a mapping $G_{B}: \mathcal{F}_{b} \rightarrow\{-,+\}$.
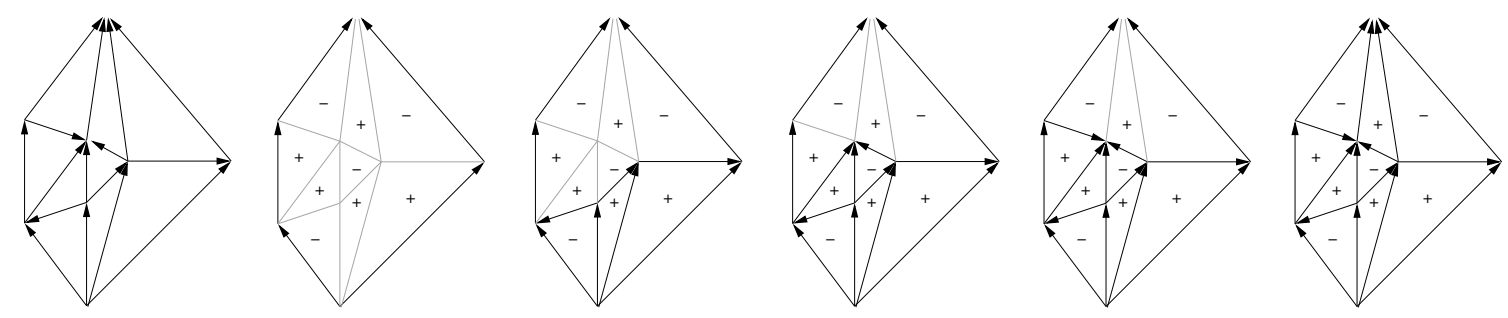

Figure 18: A bipolar orientation, the corresponding +/- encoding and an illustration of the decoding algorithm.

Theorem 12 Let $M$ be a plane inner triangulation and $B$ a bipolar orientation of $M$. Given $G_{B}$, i.e., the signs of bounded faces, it is possible to recover $B$. In other words the function $B \rightarrow G_{B}$ is injective from $\mathcal{B}(M) \rightarrow\{-,+\}^{\left|\mathcal{F}_{b}\right|}$. 
Proof. Given $G_{B}$ we construct $B$. We start by orienting all edges on the boundary of the outer face such that $s$ and $t$ are the unique source and sink of this face. We extend this partial orientation $Y$ with two rules. The vertex rule is applied to a vertex $v$, which already has incoming and outgoing edges. It takes a bundle of consecutive edges of $v$ which is bounded by two outgoing edges. It orients all the edges of the bundle such that they are outgoing at $v$. Note that these edge orientations are forced by Property $\left(1^{\prime}\right)$ of bipolar orientations in Proposition 6. The face rule is applied to a facial triangle $\Delta$, which has two oriented edges. The $\operatorname{sign} G_{B}(\Delta)$ is used to deduce the orientation of the third edge.

Note that these two rules preserve the property that every vertex $v$, which is incident to an oriented edge in $Y$, can be reached from $s$ along an oriented path. In particular $v$ has an incoming edge.

Let $A_{Y}$ be the union of all faces, which have all boundary edges oriented. Initially, $A_{Y}$ consists of the outer face. Since $B$ is acyclic the boundary of $A_{Y}$ is acyclic as well. Consequently, as long as there are faces which do not belong to $A_{Y}$, there is a vertex $v$ on the boundary of $A_{Y}$ which has two outgoing edges that belong to the boundary of $A_{Y}$. Either $v$ is a candidate to extend the orientation using the vertex rule or there is a face incident to $v$ which becomes an element of $A_{Y}$ by applying the face rule to it.

We have thus shown that the rules can be applied until $A_{Y}$ is the whole plane, i.e., all edges are oriented. They have to be oriented as in $B$, by construction.

The next theorem gives a necessary and sufficient condition for a vector in $\{-,+\}^{\left|\mathcal{F}_{b}\right|}$ to induce a bipolar orientation. For the sake of simplicity we state it only for triangulations, but the generalization to inner triangulations is straight forward. In order to obtain a more elegant formulation, we adopt the convention, that the unbounded face is signed + if the bounded face adjacent to sink and source is signed - . Otherwise the unbounded face is signed - . Thus we work now with signings of the set $\mathcal{F}$ of all faces. We say that a + triangle is the right knee of the vertex at which it has in incoming and an outgoing edge. Similarly a - triangle is the left knee of exactly one of its vertices.

Theorem 13 Let $T$ be a triangulation, $x \in\{-,+\}^{|\mathcal{F}|}$, and $\mathcal{F}^{-}$and $\mathcal{F}^{+}$the sets of faces that have negative respectively positive sign in $x$. Let $\widehat{M}(T)^{+}$and $\widehat{M}(T)^{-}$denote the subgraphs of the reduced angle graph $\widehat{M}(T)-\{s, t\}$ induced by $V \cup \mathcal{F}^{-}$respectively $V \cup \mathcal{F}^{+}$.

Then, $x$ induces a bipolar orientation on $T$ if and only if both, $\widehat{M}(T)^{+}$and $\widehat{M}(T)^{-}$, have a unique perfect matching.

Theorem 13 implies that every vertex of $T$ other than $s, t$ must be adjacent to at least one + triangle and one - triangle.

Proposition 11 Let $T$ be a plane inner triangulation. Then, T has at most $3.97^{n}$ bipolar orientations.

Proof. By Euler's formula, there are $2^{f-1} \leq 2^{2 n-2-f_{\infty}}=4^{n-1} \cdot 2^{-f_{\infty}}$ many binary vectors of length $f-1$. By the bijection from Theorem $12,4^{n-1} \cdot 2^{-f_{\infty}}$ is also an upper bound for the number of bipolar orientations of $T$. 
To squeeze the bound below $4^{n}$ we use the above observation that every vertex of $T$ must be adjacent to at least one plus + triangle and at least one - triangle. Thus, out of the $2^{d_{v}}$ possible $+/-$ vectors at a vertex $v$ at least two are not feasible. Similarly, at an outer vertex $v \neq s, t$, there is exactly one angle forming a knee at $v$. The sign of this angle depends on which of the two oriented paths of the outer boundary $v$ lies on, but it is fixed either way. Thus, out of the $2^{d_{v}-1}$ possible sign patterns at $v$ at least one is not feasible. We summarize, that at most a fraction of $\left(1-2^{1-d_{v}}\right)$ of all sign vectors is potentially feasible at every vertex but $s$ and $t$. We denote the set $V \backslash\{s, t\}$ by $V^{\prime}$ and its cardinality by $n^{\prime}$, i.e. $n^{\prime}=n-2$.

We apply Jensen's inequality, which says that for a convex function $\varphi$ the inequality $\varphi\left(\sum x_{i} / n\right) \leq\left(\sum \varphi\left(x_{i}\right)\right) / n$ holds and the inequality is reversed if $\varphi$ is a concave function. As $\log x$ is concave we obtain

$$
\log \left(\left(\prod_{v \in V^{\prime}}\left(1-2^{1-d_{v}}\right)\right)^{1 / n^{\prime}}\right)=\frac{1}{n^{\prime}} \sum_{v \in V^{\prime}} \log \left(1-2^{1-d_{v}}\right) \leq \log \left(\frac{1}{n^{\prime}} \sum_{v \in V^{\prime}}\left(1-2^{1-d_{v}}\right)\right) .
$$

By the monotonicity of the logarithm this implies

$$
\prod_{v \in V^{\prime}}\left(1-2^{1-d_{v}}\right) \leq\left(\frac{1}{n^{\prime}} \sum_{v \in V^{\prime}}\left(1-2^{1-d_{v}}\right)\right)^{n^{\prime}} .
$$

The function $2^{x}$ is convex and we apply Jensen's inequality again which yields

$$
\frac{1}{n^{\prime}} \sum_{v \in V^{\prime}}\left(1-2^{1-d_{v}}\right)=1-2\left(\frac{1}{n^{\prime}} \sum_{v \in V^{\prime}} 2^{-d_{v}}\right) \leq 1-2^{1+1 / n^{\prime} \cdot \sum_{v \in V^{\prime}}-d_{v}} .
$$

Since we deal with simple plane graphs $\sum_{v \in V^{\prime}} d_{v} \leq 2(3 n-6)=6 n^{\prime}$ and we conclude

$$
\prod_{v \in V^{\prime}}\left(1-2^{1-d_{v}}\right) \leq\left(1-2^{1-6}\right)^{n^{\prime}}=\left(\frac{31}{32}\right)^{n^{\prime}} .
$$

Since we deal with plane graphs, $T$ can be partitioned into at most four independent sets $I_{k}, k=1, \ldots, 4$ by the Four Color Theorem. Thus,

$$
\left(\frac{31}{32}\right)^{n^{\prime}} \geq \prod_{v \in V^{\prime}}\left(1-2^{1-d_{v}}\right)=\prod_{k=1}^{4} \prod_{v \in I_{k}}\left(1-2^{1-d_{v}}\right)
$$

and for at least one of the independent set it must hold that

$$
\left(\frac{31}{32}\right)^{n^{\prime} / 4} \geq \prod_{v \in I_{k}}\left(1-2^{1-d_{v}}\right)
$$

We are ready to conclude that there are at most

$$
4^{n-1} \cdot 2^{-f_{\infty}} \cdot\left(\frac{31}{32}\right)^{(n-2) / 4}<3.97^{n} \cdot 2^{-f_{\infty}} \cdot\left(\frac{32}{31}\right)^{1 / 2}<3.97^{n}
$$

bipolar orientations of $T$. 


\section{Complexity of Counting $\alpha$-Orientations}

Given a planar map $M$ and some $\alpha: V \rightarrow \mathbb{N}$, what is the complexity of computing the number of $\alpha$-orientations of $M$ ? In some instances this number can be computed efficiently, e.g. for perfect matchings and spanning trees of general planar maps. In Section 6.1 we show that counting is \#P-complete for other $\alpha$-orientations, and in Section 6.2 we discuss how to adapt an existing fully polynomial randomized approximation scheme.

\section{1 \#P-Completeness}

Recently, Creed [8] has shown the following theorem. As already mentioned Eulerian orientations are $\alpha$-orientations and hence Theorem 14 says that counting $\alpha$-orientations is \#P-complete. Since we use the proof technique in the sequel, we sketch the proof here. It uses a reduction from counting Eulerian orientations, which has been proven to be \#P-complete by Mihail and Winkler in [26].

Theorem 14 It is \#P-complete to count Eulerian orientations of planar graphs.

Proof. We aim to show that the number of Eulerian orientations of a graph $G$ can be computed in polynomial time with the aid of polynomially many calls to an oracle for the number of Eulerian orientations of a planar graph.

In order to count the Eulerian orientations of a graph $G$ with $n$ vertices a drawing of this graph in the plane with $\ell$ crossings is produced. We may assume that no three edges cross in the same point and that $\ell$ is of order $O\left(n^{2}\right)$.

From this drawing a family of graphs $G_{i}$ for $i=0, \ldots, \ell$ is produced. In $G_{i}$ every crossing of two edges $\{u, v\}$ and $\{x, y\}$ is replaced by the crossover box $H_{i}$ on $4 k+1$ vertices, see Figure 19. For example in $G_{0}$ every crossing is replaced by just one vertex. We call the edges $\left\{v, w_{k}^{u}\right\},\left\{u, w_{k}^{v}\right\},\left\{x, w_{k}^{x}\right\},\left\{y, w_{k}^{y}\right\}$ the connection edges of $H_{k}$.

Every Eulerian orientation of $G$ induces a Eulerian orientation of $G_{0}$, but there are Eulerian orientations of $G_{0}$, which do not come from a Eulerian orientation of $G$. Given a Eulerian orientation of $G_{0}$ we call the orientation of the edges incident to a vertex $w_{0}$, which replaces a crossing, valid if exactly one of the edges $\left\{v, w_{0}\right\},\left\{u, w_{0}\right\}$ is directed away from $w_{0}$, and invalid otherwise.
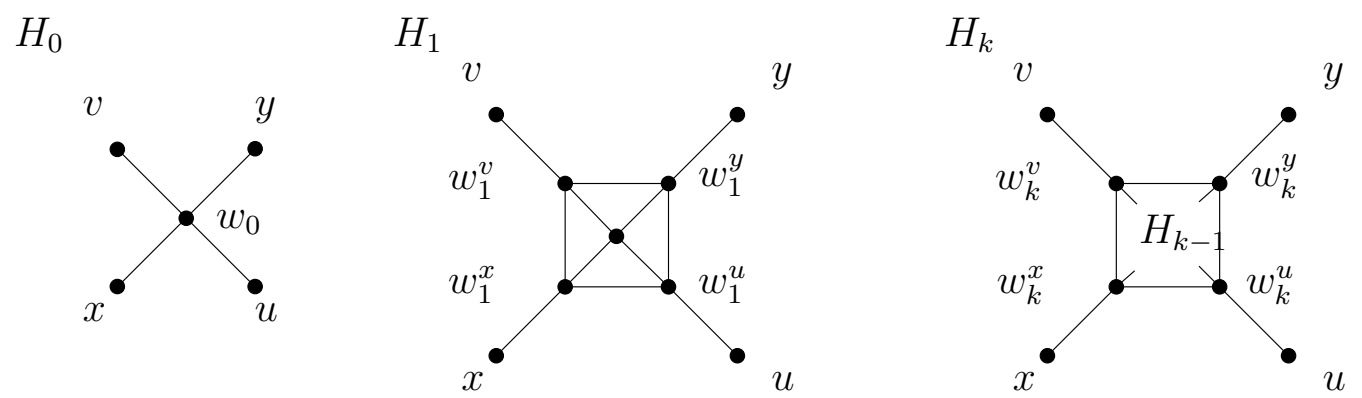

Figure 19: The crossover boxes defined in [8]. 
We call a configuration of the connection edges of $H_{k}$ valid if exactly one of the edges $\left\{v, w_{k}^{v}\right\},\left\{u, w_{k}^{u}\right\}$ is directed towards $\{u, v\}$ and exactly one of the edges $\left\{x, w_{k}^{x}\right\},\left\{y, w_{k}^{y}\right\}$ is directed towards $\{x, y\}$. We call a configuration of the connection edges of $H_{k}$ invalid if exactly two of the connection edges are directed towards $\{u, v, x, y\}$, but it is not a valid configuration. By $x_{k}$ respectively $y_{k}$ we denote the number of ways that a valid respectively invalid configuration of the connections edges of $H_{k}$ can be extended to an orientation of the edges of $H_{k}$, such that all vertices of $H_{k}$ have out-degree 2.

It is clear that $x_{0}=1=y_{0}$ and Creed observes the following recursion

$$
\begin{aligned}
& x_{k}=4 x_{k-1}+2 y_{k-1} \\
& y_{k}=4 x_{k-1}+3 y_{k-1} .
\end{aligned}
$$

This recursion formula can be verified by a simple enumeration. In [8] a lemma from [39] is used to argue that the sequence $x_{k} / y_{k}$ is non-repeating. In Lemma 5 we provide an easy argument to show from first principles that $x_{k} / y_{k}$ is strictly monotonically decreasing.

Let $N_{i}$ denote the number of Eulerian orientations of $G_{0}$ which have $i$ valid cross over boxes, that is $N_{\ell}$ is the number of Eulerian orientations of $G$. The number of Eulerian orientations of $G_{k}$ is

$$
E O\left(G_{k}\right)=\sum_{i=0}^{\ell} N_{i} x_{k}^{i} y_{k}^{\ell-i}
$$

Hence the number $E O\left(G_{k}\right) / y_{k}^{\ell}$ is the value of the polynomial $p(z)=\sum_{i=0}^{\ell} N_{i} z^{i}$ at the point $x_{k} / y_{k}$. Since computing $y_{k}^{\ell}$ is easy the polynomial $p$ of degree $\ell$ can be evaluated at $\ell+1$ different points with $\ell+1$ calls to an oracle for counting Eulerian orientations of planar graphs. Hence the coefficients of $p$ can be determined using polynomial interpolation. In particular this yields a way to compute $N_{\ell}$.

Lemma 5 Let $x_{0}=1=y_{0}$ and $x_{k+1}=4 x_{k}+2 y_{k}$ while $y_{k+1}=4 x_{k}+3 y_{k}$. Then the sequence $x_{k} / y_{k}$ is strictly monotonically decreasing.

Proof. It follows directly from the recursion that $x_{k+1}=y_{k+1}-y_{k}$ and $y_{k+1}=7 y_{k}-4 y_{k-1}$. We want to show that

$$
\frac{x_{k+1}}{y_{k+1}}=\frac{4 x_{k}+2 y_{k}}{4 x_{k}+3 y_{k}}<\frac{x_{k}}{y_{k}} \Longleftrightarrow 4\left(\frac{x_{k}}{y_{k}}\right)^{2}-\frac{x_{k}}{y_{k}}-2>0
$$

Since $x_{k} / y_{k}>0$ this inequality is satisfied if and only if $x_{k} / y_{k}>(1+\sqrt{33}) / 8=: c$. Note that $1 /(1-c)=3+4 c$ can be easily derived since $c$ solves $4 t^{2}-t-2=0$.

It remains to show that $x_{k} / y_{k}=1-\left(y_{k-1} / y_{k}\right)>c$, which we do by induction. Since $x_{0} / y_{0}=1$ and $x_{1} / y_{1}>6 / 7$ the induction base is trivial and the induction hypothesis guarantees that $1-c>y_{k-2} / y_{k-1}$ for $k \geq 2$. We have

$$
\frac{x_{k}}{y_{k}}=1-\frac{y_{k-1}}{y_{k}}>c \Longleftrightarrow \frac{y_{k}}{y_{k-1}}>\frac{1}{1-c} .
$$


Using the induction hypothesis for the last inequality we obtain that

$$
\frac{y_{k}}{y_{k-1}}=\frac{7 y_{k-1}-4 y_{k-2}}{y_{k-1}}=7-4 \frac{y_{k-2}}{y_{k-1}}>3+4 c=\frac{1}{1-c}
$$

The reduction from counting perfect matchings in bipartite graphs in [26] to counting Eulerian orientations creates vertex degrees, which grow linearly with the number of vertices of the graph. This implies also that the image of $\alpha(v)=d(v) / 2$ grows with $n$. The following theorem shows that this dependence of $\alpha$ on $n$ is not necessary to obtain a \#P-completeness result.

Theorem 15 For the following instances of graph classes and out-degree functions $\alpha$ the counting of $\alpha$-orientations is \#P-complete.

1. Planar maps with $d(v)=4$ and $\alpha(v) \in\{1,2,3\}$ for all $v \in V$.

2. Planar maps with $d(v) \in\{3,4,5\}$ and $\alpha(v)=2$ for all $v \in V$.

The proof uses the planarization method from the proof of Theorem 14 in conjunction with the following theorem from [9].

Theorem 16 Counting perfect matchings of k-regular bipartite graphs is \#P-complete for every $k \geq 3$.

Proof of Theorem 15. Perfect matchings of a bipartite graph $G$ with vertex set $V=A \cup B$ are in bijection with $\alpha$-orientations of $G$ with $\alpha(v)=1$ for $v \in A$ and $\alpha(v)=d(v)-1$ for $v \in B$. This bijection is established by identifying matchings edges with edges directed from $A$ to $B$. Hence in $k$-regular bipartite graphs counting perfect matchings is equivalent to counting what we call 1 - $(k-1)$-orientations in the sequel.

We observe that the planarization method from the proof of Theorem 14 can be used in a more general setting. Let $\mathcal{G}_{D}$ be the set of all graphs with vertex degrees in $D \subset \mathbb{N}$ and $\mathcal{P}_{D}$ the set of all planar graphs with degrees in $D$. Let $I \subset \mathbb{N}$ and associate with every $G \in \mathcal{G}_{D}$ an out-degree function $\alpha_{G}$ whose image is contained in $I$. Then, the proof of Theorem 14 shows that counting the $\alpha_{G}$-orientations of graphs in $\mathcal{G}_{D}$ can be reduced to counting $\alpha_{G}^{\prime}$-orientations of the graphs in $\mathcal{P}_{D \cup\{4\}}$ where the image of $\alpha_{G}^{\prime}$ is contained in $I \cup\{2\}$ for all $G^{\prime} \in \mathcal{P}_{D \cup\{4\}}$.

When we apply this to 4-regular bipartite graphs with 1-3-orientations, that is perfect matchings, it yields the first claim of the theorem since counting perfect matchings of bipartite 4-regular graphs is \#P-complete by Theorem 16. We give two different proofs for the second claim.

Let $G$ be a graph with a degree 3 vertex $v$ and $\alpha_{G}$ an associated out-degree function with $\alpha_{G}(v)=1$. We substitute $v$ by the gadget $G_{1}$ from Figure 20 (a) to obtain a graph $G^{\prime}$. The gadget has five vertices that induce nine edges and 3 edges connect it with the neighbors of $v$ in $G$. Let $\alpha_{G^{\prime}}=\alpha_{G}$ on $V(G)-v$ and be $\alpha_{G^{\prime}}(u)=2$ for $u \in V\left(G_{1}\right)$. Exactly 
(a)

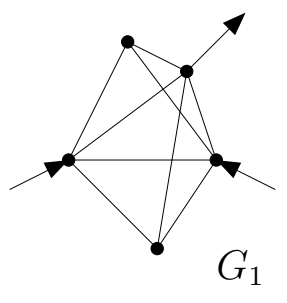

(b)

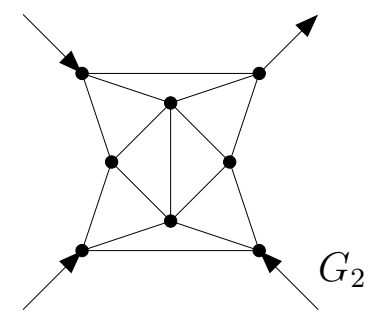

(c)

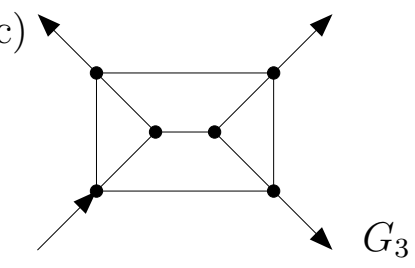

Figure 20: Gadgets to translate (a) $\alpha(v)=1$ to $\alpha \equiv 2$ for $d(v)=3$, (b) $\alpha(v)=1$ to $\alpha \equiv 2$ for $d(v)=4$, and (c) $\alpha(v)=3$ to $\alpha \equiv 2$ for $d(v)=4$.

one of the connection edges must be directed away from $G_{1}$ in every $\alpha_{G^{\prime}}$-orientation of $G^{\prime}$. Note that $G_{1}$ is symmetric in the three connection vertices. It is easy to check that $G_{1}$ has ten orientations with out-degree 2 at every vertex once the outgoing connection edge has been chosen. Thus, every $\alpha_{G^{-}}$orientation is associated with ten $\alpha_{G^{-}}^{\prime}$ orientations of $G^{\prime}$ and since the underlying $\alpha_{G^{-}}$-rientations can be reconstructed from every $\alpha_{G^{\prime}}$-orientation we obtain that $r_{\alpha_{G^{\prime}}}\left(G^{\prime}\right)=10 \cdot r_{\alpha_{G}}(G)$.

Let a 3-regular bipartite graph $G$ with vertex set $V=A \cup B$ be given and $G^{\prime}$ be obtained from $G$ by substituting every vertex $v \in A$ by a copy of $G_{1}$. The number of 1-2-orientations of $G$ is $10^{-|A|} \cdot r_{\alpha_{G^{\prime}}}\left(G^{\prime}\right)$. Using the planarization method this yields that counting 2-orientations of graphs from $\mathcal{P}_{\{3,4,5\}}$ is \#P-complete.

Similarly counting perfect matchings of 4-regular bipartite graphs can be reduced to counting 2-orientations of graphs from $\mathcal{P}_{\{3,4,5\}}$. We mention this second proof since it uses planar gadgets. More precisely $G^{\prime}$ is obtained from $G$ by substituting the vertices of one partition class of a 4-regular bipartite graph $G$ by the gadget $G_{2}$ shown in Figure 20 (b) and the vertices from the other partition class by the gadget $G_{3}$ shown in Figure 20 (c). Using a similar reasoning as above one obtains that the number of 1-3-orientations of $G$ is $(26 \cdot 6)^{-|A|} r_{\alpha_{G^{\prime}}}\left(G^{\prime}\right)$, where $G_{2}$ and $G_{3}$ give a blow up factor 26 respectively 6 .

Having proved Theorem 15 it is natural to ask whether it is \#P-complete to count $\alpha$-orientations for $k$-regular planar graphs and constant $\alpha$. This setting implies that $\alpha \equiv k / 2$. Planar graphs have average degree less than six. Furthermore a 2-regular connected graph, that is a cycle, has two Eulerian orientations. Hence the above question should be asked for $k=4$. Note, that the planarization method yields vertices with $d(v)=4$ and $\alpha(v)=2$, so we do not need to restrict our considerations to planar graphs when trying to answer this question.

Problem. Is it \#P-complete to count Eulerian orientations of 4-regular graphs?

To the best of our knowledge even the following problem is also open.

Problem. Is it \#P-complete to count Eulerian orientations of graphs with degrees in $\{1, \ldots, k\}$, for some arbitrary but fixed $k \in \mathbb{N}$ ?

We present one more \#P-completeness result since it has a nice connection with the first problem stated above. Note, that for 4-regular bipartite graphs 2-factors and Eulerian orientations are in bijection. For 3-regular bipartite graphs 2-factors are in bijection with 
their complements the 1-factors, i.e. perfect matchings. Hence it is \#P-complete to count 2 -factors of 3-regular bipartite graphs. The next theorem generalizes this observation.

Theorem 17 For every $i \geq 3, i \neq 4$ counting 2-factors of $i$-regular bipartite graphs is \#P-complete.

Proof. The case $i=3$ follows from Theorem 16 as explained above. The proof for $i \geq 5$ is a reduction from counting 2-factors of 3-regular bipartite graphs. The method comes from the proof of Theorem 16 in [9].

The following preliminary considerations will be needed later. We fix some edge $e_{0}$ of the complete bipartite graph on $2 i$ vertices $K_{i, i}$. This graph has $i^{2}$ edges and every 2 -factor of $K_{i, i}$ has $2 i$ edges. Let $c_{i}$ be the number of 2 -factors of $K_{i, i}$. We want to find the ratio between 2 -factors of $K_{i, i}$ which contain $e_{0}$ and 2 -factors which do not contain $e_{0}$. Consider pairs of 2-factors and edges $(F, e)$. Obviously, there are $i^{2} \cdot c_{i}$ such pairs and we have that $|\{(F, e) \mid e \in F\}|=2 i \cdot c_{i}$ while $|\{(F, e) \mid e \notin F\}|=\left(i^{2}-2 i\right) \cdot c_{i}$. It is obvious that

$$
\{(F, e) \mid e \in F\}=\bigcup_{j=1}^{|E|}\left\{\left(F, e_{j}\right) \mid e_{j} \in F\right\}
$$

and symmetry implies, that all sets $\left\{\left(F, e_{j}\right) \mid e_{j} \in F\right\}$ have the same cardinality. We conclude, that there are $a_{i}=2 c_{i} / i$ 2-factors including $e_{0}$. It follows similarly, that there are $b_{i}=(1-2 / i) c_{i} 2$-factors not including $e_{0}$. We infer that $a_{i} / b_{i}=2 /(i-2) \leq 2 / 3$ for $i \geq 5$.

The gadget $P_{i}(k)$ is a concatenation of $k$ disjoint copies of $K_{i, i}-e_{0}$, with $k-1$ connection edges as shown in Figure 21 for $i=5$ and $k=4$. The gadget is connected to the rest of the graph via two edges at the degree $i-1$ vertices.

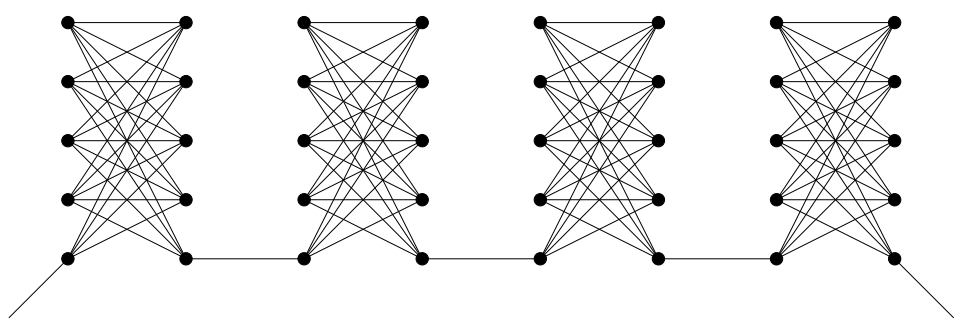

Figure 21: The bridge gadget $P_{5}(4)$

Let $G$ be a 3-regular bipartite graph with $2 n$ vertices and $G^{\prime}(k)$ be obtained from $G$ by augmenting it with $n(i-3)$ disjoint bridge gadgets $P_{i}(k)$ such that $G^{\prime}(k)$ is $i$-regular. Let $P$ be some fixed bridge. Note that every 2-factor of $G^{\prime}(k)$ includes all or none of the connection edges of $P$. This is because a 2 -factor is a partition into cycles and therefore intersects every edge cut of cardinality two in either none or both of the edges.

Let $c_{G}$ be the number of 2-factors of $G$. Among the 2-factors of $G^{\prime}(k)$ let $S$ denote the set of those, which induce a 2-factor of $G$, and $S^{c}$ the other 2-factors. We have $|S|=c_{G} \cdot b_{i}^{k \cdot n(i-3)}$ since the 2-factors in $S$ cannot include any connection edges and thus 
can be partitioned into a 2-factor of $G$ which is augmented by one of the $b_{i}^{k}$ possible 2 -factors in every bridge.

Next we want an upper bound for $\left|S^{c}\right|$. Every 2-factor in $S^{c}$ includes the connection edges of at least one bridge $P$. Since $2^{3 n}$ is the number of subsets of $E(G)$ each of which can be augmented to a 2-factor in at most $a_{i}^{k}$ ways on $P$ we have that $\left|S^{c}\right| \leq a_{i}^{k} b_{i}^{k \cdot(n(i-3)-1)} 2^{3 n}$. The number of 2-factors of $G^{\prime}(k)$ is $c_{G^{\prime}(k)}=|S|+\left|S^{c}\right|$ for which we have the following bounds.

$$
\begin{aligned}
c_{G} \cdot b_{i}^{k \cdot n(i-3)} & \leq c_{G^{\prime}(k)} \\
\Longleftrightarrow c_{G} & \leq c_{G^{\prime}(k)} b_{i}^{-k \cdot n(i-3)} \leq b_{i}^{k \cdot n(i-3)}+a_{i}^{k} b_{i}^{k \cdot(n(i-3)-1)} 2^{3 n} \\
& \leq c_{G}+2^{3 n}\left(\frac{a_{i}}{b_{i}}\right)^{k}
\end{aligned}
$$

Since $a_{i} / b_{i} \leq 2 / 3$ we have that

$$
2^{3 n}\left(\frac{a_{i}}{b_{i}}\right)^{k}<\frac{1}{2} \text { for } k>3 /\left(\log _{2} 3-1\right) n+1 /\left(\log _{2} 3-1\right) .
$$

Note that the lower bound for $k$ is linear in $n$.

We finally conclude that $c_{G}=\left\lfloor c_{G^{\prime}} b_{i}^{-k \cdot n(i-3)}\right\rfloor$ for $k$ large enough. Since $b_{i}^{-k \cdot n(i-3)}$ is easy to compute and $G_{k}$ has size polynomial in $n$ this proves the theorem.

Remark. We would like to point out, that the missing case $k=4$ in Theorem 17 cannot be fixed by substituting the bridge gadget by another gadget. It is crucial that $a_{i} / b_{i}<1$ and symmetry implies, that in every 4-regular graph the number of 2 -factors including a fixed edge $e_{0}$ is equal to the number of 2 -factors not containing $e_{0}$.

\subsection{Approximation}

Let $f: V \rightarrow \mathbb{N}$ be defined on the vertex set of a graph $G$. An $f$-factor of a graph $G$ is a subgraph of $G$ such that every vertex has degree $f(v)$ in $F$.

Counting $\alpha$-orientations can be reduced to counting $f$-factors in bipartite planar graphs and to counting perfect matchings in bipartite graphs. We next describe these transformations. They are useful because bipartite perfect matchings have been the subject of extensive research (for example [24, 29, 20]).

First, note that the $\alpha$-orientations of $M$ are in bijection with the the $\alpha^{\prime}$-orientations of the bipartite planar map $M^{\prime}$ obtained from $M$ by subdividing every edge once. Here $\alpha^{\prime}(v)=\alpha(v)$ for the original vertices of $M$ and $\alpha^{\prime}(v)=1$ for all subdivision vertices. The $\alpha^{\prime}$-orientations of $M^{\prime}$ are in bijection with the $f$-factors of $M^{\prime}$ where $f(v)=\alpha^{\prime}(v)$ for all vertices of $M^{\prime}$. The bijection works by identifying factor edges with edges directed from a vertex of $M$ to an edge vertex.

The idea for the next transformation is due to Tutte [37]. The graph $M^{\prime}$ gets blown up into a graph $M^{\prime \prime}$ such that $M^{\prime \prime}$ has $\prod_{v \in M}(d(v)-f(v))$ ! times as many perfect matchings, i.e., 1-factors, as there are $f$-factors of $M^{\prime}$. To obtain $M^{\prime \prime}$ from $M^{\prime}$ substitute $v \in V(M)$ by a $K_{d(v), d(v)-f(v)}$, such that each of the $d(v)$ edges incident to $v$ in $M^{\prime}$ connects to one of the vertices from the partition class of cardinality $d(v)$. 
In [20] Jerrum, Sinclair, and Vigoda give a fully polynomial randomized approximation scheme for counting perfect matchings of bipartite graphs. Thus, the above transformation yields a fully polynomial randomized approximation scheme for $\alpha$-orientations as well.

The number of perfect matchings of a bipartite graph with a Pfaffian orientation can be computed in polynomial time. Little [23] gave a full characterization of graphs with a Pfaffian orientation and in [29] a polynomial time algorithm to test whether a given graph is Pfaffian is introduced. As a special case of Little's characterizations it follows that all planar graphs are Pfaffian. Hence, in all cases where $M^{\prime \prime}$ is planar the counting is easy. For spanning trees the above transformations yields planar graphs, while for Eulerian orientations it does not, as Theorem 14 implies. Although we do not have a hardness result for Schnyder woods and bipolar orientations on planar maps, there are in both cases instances for which the transformation yields a non-Pfaffian graph. One such example is the augmented triangular grid from Section 3.1. Figure 22 shows a local structure with five vertices and four faces that implies that $M^{\prime \prime}$ is not Pfaffian. The figure shows simplified versions of $\widehat{M}^{\prime}$ and $\widehat{M}^{\prime \prime}$. We can choose $\widehat{M}^{\prime}=\widehat{M}$ since the angle graph is bipartite. The Tutte transformation substitutes face vertices by $K_{1,3}$ and primal vertices of degree $d$ by $K_{2, d}$. Instead one can simply create a copy of every primal vertex with the same neighborhood as the original and leave the face vertices unchanged to obtain a simplified version of $\widehat{M}^{\prime \prime}$.
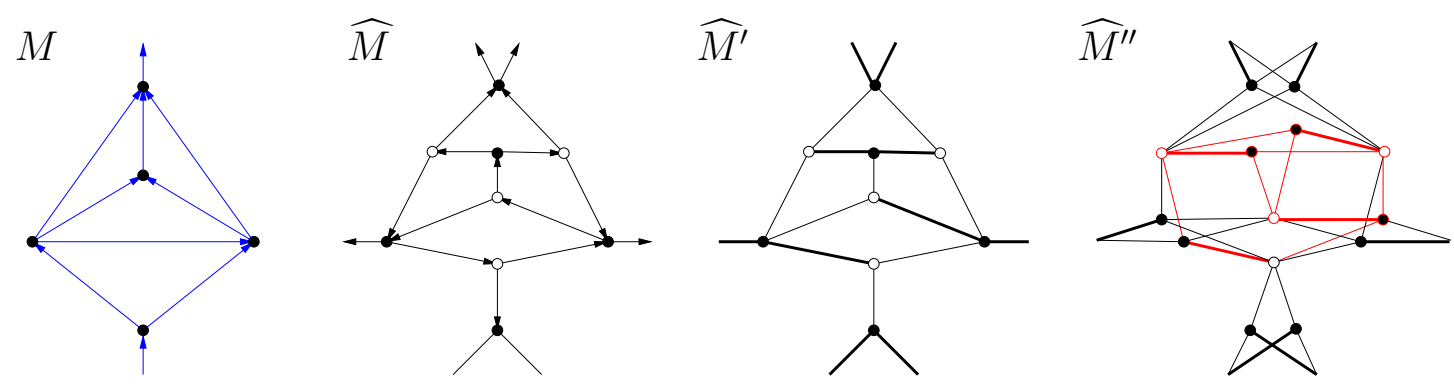

Figure 22: An oriented subgraph of a triangulation with a bipolar orientation that induces a central $K_{3,3}$-subdivision.

\section{Conclusions}

In this paper we have studied the maximum number of $\alpha$-orientations for different classes of planar maps and different $\alpha$. In most cases we have exponential upper and lower bounds $c_{L}^{n}$ and $c_{U}^{n}$ for this number.

The obvious problem is to improve on the constants $c_{L}$ and $c_{U}$ for the different instances. We think, that in particular improving the upper bound of $8^{n}$ for the number of Schnyder woods on 3-connected planar maps is worth further efforts. For bipolar orientations the more efficient encoding from Theorem 12 helps to improve the upper bound. We think that finding a more efficient encoding for Schnyder woods might be needed to substantially improve on the $8^{n}$ bound. 
Results by Lieb [22] and Baxter [1] yield the exact asymptotic behavior of the number of Eulerian orientations for the square and triangular grid on the torus. This yields upper bounds for the number of 2-orientations on the square grid and the number of Schnyder woods on the triangular grid. We have not been able to take advantage of these results for improving the lower bounds for the number of 2-orientations respectively Schnyder woods.

For some instance of $\alpha$-orientations there are \#P-completeness results. This contrasts with spanning trees and planar bipartite perfect matchings for which polynomial algorithms are available. It remains open to determine the complexity of counting Schnyder woods and bipolar orientations on planar maps and of counting Eulerian orientations of graphs with bounded maximum degree.

Acknowledgments. We would like to thank Graham Brightwell for interesting discussions and valuable hints in connection with Lieb's 3-coloring of the square grid. We thank Christian Krattenthaler for directing us to reference [21] and Mark Jerrum for bringing Páidí Creed's work to our attention. Thanks to Paídí Creed for sending us a preliminary version of his proof. Florian Zickfeld was supported by the Studienstiftung des deutschen Volkes.

\section{References}

[1] R. J. BAxter, F model on a triangular lattice, J. Math. Physics, 10 (1969), pp. 12111216.

[2] R. J. BAxter, Exactly solved models in statistical mechanics, Academic Press, 1982.

[3] N. Bonichon, A bijection between realizers of maximal plane graphs and pairs of non-crossing dyck paths, Discrete Mathematics, 298 (2005), pp. 104-114. FPSAC'02 Special Issue.

[4] N. Bonichon, S. Felsner, And M. Mosbah, Convex drawings of 3-connected planar graphs, in Graph Drawing (Proc. GD '04), vol. 3383 of LNCS, 2004, pp. 6070 .

[5] P. S. Bonsma, Spanning trees with many leaves in graphs with minimum degree three. Submitted to SIAM Journal on Discrete Mathematics.

[6] P. S. Bonsma, Sparse Cuts, Matching-Cuts and Leafy Trees in Graphs, PhD thesis, University of Twente, 2006.

[7] N. J. CALKIN AND H. S. Wilf, The number of independent sets in a grid graph, SIAM J. Discrete Math., 11 (1998), pp. 54-60.

[8] P. Creed, Counting Eulerian orientations is planar graphs is \#P-complete. Personal Communication, 2007.

[9] P. Dagum And M. Luby, Approximating the permanent of graphs with large factors, Theoretical Computer Science, 102 (1992), pp. 283-305. 
[10] H. DE Fraysseix And P. O. DE Mendez, On topological aspects of orientation, Discrete Math., 229 (2001), pp. 57-72.

[11] H. de Fraysseix, P. O. De Mendez, And P. Rosenstiehl, Bipolar orientations revisited, Discrete Appl. Math., 56 (1995), pp. 157-179.

[12] S. FELSNER, Convex drawings of planar graphs and the order dimension of 3polytopes, Order, 18 (2001), pp. 19-37.

[13] S. Felsner, Geometric Graphs and Arrangements, Vieweg Verlag, 2004.

[14] S. FELSNER, Lattice structures from planar graphs, Elec. J. Comb., (2004). R15.

[15] S. Felsner, É. Fusy, M. Noy, D. Orden, D. Poulalhon, and G. SchaefFER, Baxter families and more: Bijections and counting, 2007. in preparation.

[16] S. Felsner, C. Huemer, S. Kappes, and D. Orden, Binary labelings for plane quadrangulations and their relatives. preprint 2007.

[17] É. FUsy, Transversal structures on triangulations, with application to straight-line drawing., in Graph Drawing(Proc. GD '05), 2005, pp. 177-188.

[18] É. Fusy, Combinatorics of Plane Maps with Algorithmic Applications, PhD thesis, École Polytechnique, 2007.

[19] É. Fusy, D. Poulalhon, And G. Schaeffer, Dissections and trees, with applications to optimal mesh encoding and to random sampling, in SODA, 2005, pp. 690-699.

[20] M. Jerrum, A. Sinclair, And E. VigodA, A polynomial-time approximation algorithm for the permanent of a matrix with non-negative entries, JACM, 51 (2004), pp. 671-697.

[21] R. W. Kenyon, J. G. Propp, And D. B. Wilson, Trees and matchings, Elec. J. Comb., 7 (2000).

[22] E. H. LiEB, The residual entropy of square ice, Physical Review, 162 (1967), pp. 162172.

[23] C. H. C. LitTle, A characterization of convertible $(0,1)$-matrices, J. Combin. Theory Ser. B, 18 (1975), pp. 187-208.

[24] L. Lovász And M. D. Plummer, Matching Theory, no. 29 in Annals of Discrete Mathematics, North-Holland, 1986.

[25] S. Melang, Bipolare Orientierungen planarer Graphen, master's thesis, Technische Universität Berlin, 2006.

[26] M. Mihail And P. Winkler, On the nubmer of Eulerian orienations of a graph, Algorithmica, 16 (1996), pp. 402-424.

[27] D. Poulalhon And G. Schaeffer, Optimal coding and sampling of triangulations, in Proceedings of ICALP'03, vol. 2719 of LNCS, Springer, 2003, pp. 1080-1094.

[28] A. RiBó, Realization and Counting Problems for Planar Structures: Trees and Linkages, Polytopes and Polyominoes, PhD thesis, Freie Unversität Berlin, 2006.

[29] N. Robertson, P. D. Seymour, and R. Thomas, Permanents, Pfaffian orientations, and even directed circuits, Ann. of Math., 150 (1999), pp. 929-975. 
[30] P. Rosenstienl, Embedding in the plane with orientation constraints: the angle graph, Ann. New York Acad. Sci., (1983), pp. 340-346.

[31] G. Rote, The number of spanning trees in a planar graph, in Oberwolfach Reports, vol. 2, EMS, 2005, pp. 969-973. http://page.mi.fu-berlin.de/rote/about_me/ publications.html.

[32] W. Schnyder, Planar graphs and poset dimension, Order, 5 (1989), pp. 323-343.

[33] W. SchNYDER, Embedding planar graphs on the grid, Proc. 1st ACM-SIAM Sympos. Discrete Algorithms, 5 (1990), pp. 138-148.

[34] N. J. A. SloAne, The on-line encyclopedia of integer sequences. http://www. research.att.com $/ \sim n j a s /$ sequences.

[35] R. Tamassia and I. G. Tollis, A unified approach to visibility representations of planar graphs, Discrete Comput. Geom., 1 (1986), pp. 321-341.

[36] H. N. V. Temperley, Enumeration of graphs on a large periodic lattice, in Combinatorics: Proceedings of the British Combinatorial Conference 1973, London Mathematical Society Lecture Note Series $\sharp 13,1974$, pp. 155-159.

[37] W. T. TutTe, A short proof of the factor theorem for finite graphs, Canadian Journal of Mathematics, 6 (1954), pp. 347-352.

[38] W. T. TutTe, A census of planar triangulations, Canadian Journal of Mathematics, 14 (1962), pp. 138-148.

[39] S. P. VADHAN, The complexity of counting in sparse, regular, and planar graphs, SIAM Journal of Computing, 31 (2001), pp. 398-427.

[40] D. R. Woods, Drawing Planar Graphs, PhD thesis, Stanford University, 1982. Technical Report STAN-CS-82-943. 